\title{
Safety of indacaterol in the treatment of patients with COPD
}

This article was published in the following Dove Press journal:

International Journal of COPD

2I September 201 I

Number of times this article has been viewed

\author{
James F Donohue' \\ Dave Singh ${ }^{2}$ \\ Oliver Kornmann ${ }^{3}$ \\ David Lawrence ${ }^{4}$ \\ Cheryl Lassen ${ }^{4}$ \\ Benjamin Kramer ${ }^{5}$ \\ 'University of North Carolina, \\ Chapel Hill, NC, USA; ${ }^{2}$ University \\ of Manchester, Medicines Evaluation \\ Unit, Manchester, UK; ${ }^{3} \mathrm{KF}$ \\ Pneumologie $\mathrm{GmbH}$ and Co KG. \\ Clinical Research Centre Respiratory \\ Diseases, Frankfurt, Germany; \\ ${ }^{4}$ Novartis Horsham Research \\ Centre, Horsham, UK; ${ }^{5}$ Novartis \\ Pharmaceuticals Inc, East Hanover, \\ New Jersey, USA
}

Correspondence: James F Donohue University of North Carolina, 4125 Biolnformatics Building, I 30 Mason Farm Road, CB 7020, Chapel Hill,

NC, 27599, USA

$\mathrm{Tel}+\mathrm{I} 9199662531$

Fax +19199667013

Email james_donohue@med.unc.edu
Purpose: Pooled data were analyzed to evaluate the safety and tolerability of indacaterol, a once-daily inhaled long-acting $\beta_{2}$-agonist for chronic obstructive pulmonary disease (COPD). Patients and methods: Data were pooled from clinical studies of 3-12 months' duration in patients with moderate-to-severe COPD receiving double-blind indacaterol $75 \mu \mathrm{g}(\mathrm{n}=449)$, $150 \mu \mathrm{g}(\mathrm{n}=2611), 300 \mu \mathrm{g}(\mathrm{n}=1157)$, or $600 \mu \mathrm{g}$ once daily $(\mathrm{n}=547)$; formoterol $12 \mu \mathrm{g}$ twice daily $(\mathrm{n}=556)$; salmeterol $50 \mu \mathrm{g}$ twice daily $(\mathrm{n}=895)$; placebo $(\mathrm{n}=2012)$; or tiotropium $18 \mu \mathrm{g}$ once daily, given open label or blinded $(n=1214)$. Outcomes were adverse events, serious adverse events and deaths, plasma potassium, blood glucose, and QTc interval and vital signs.

Results: The commonest adverse events with indacaterol were COPD worsening, nasopharyngitis, and headache; most cases were mild or moderate and incidence was generally similar to placebo and other active treatments. The risk of acute respiratory serious adverse events (leading to hospitalization, intubation, or death) was not significantly increased with any of the active treatments compared with placebo. COPD exacerbation rates (analyzed in the intent-to-treat population) were significantly reduced with all active treatments versus placebo. Hazard ratios versus placebo for major cardiovascular adverse events were $<1$ for all indacaterol doses. Notable values for vital signs and measures of systemic $\beta_{2}$-adrenoceptor activity were rare with indacaterol. The number of deaths adjusted per patient-year was lower with indacaterol (all doses combined) than with placebo (relative risk 0.21 [95\% confidence interval 0.07-0.660], $P=0.008$ ).

Conclusion: Indacaterol has a good profile of safety and tolerability that is appropriate for the maintenance treatment of patients with COPD.

Keywords: indacaterol, safety, tolerability, formoterol, salmeterol, tiotropium

\section{Introduction}

Regular maintenance treatment with one or more long-acting bronchodilators is recommended for patients with moderate or more severe chronic obstructive pulmonary disease (COPD) to provide improved symptom control beyond that achieved with short-acting bronchodilators alone. ${ }^{1}$ Two classes of long-acting inhaled bronchodilators are currently available, the once-daily anticholinergic, tiotropium, and the long-acting $\beta_{2}$-agonists (LABAs), taken either twice daily (formoterol and salmeterol) or once daily (indacaterol).

Clinical studies with indacaterol have reported 24-hour bronchodilator efficacy and improved clinical outcomes in the form of reduced symptoms and better health status. These studies also reported reassuring safety and tolerability data with doses up to and including $600 \mu \mathrm{g}$ once daily, double the highest licensed dose, given for up to 
1 year. $^{2-6}$ As the first once-daily inhaled $\beta_{2}$-agonist licensed for the treatment of COPD, it is relevant to scrutinize the safety of indacaterol, especially when many patients with COPD are elderly and comorbid conditions are common. ${ }^{7-9}$ Here, an analysis of pooled safety data from recent clinical trials with indacaterol, providing a large patient population in which potential safety signals can be more robustly and comprehensively assessed compared with single studies, is reported. This pooled data set allows the safety of indacaterol to be compared with placebo and with data from formoterol, salmeterol, and tiotropium treatment groups.

\section{Methods}

This evaluation of safety data from the indacaterol clinical development program includes data from all completed indacaterol studies of at least 12 weeks in duration in COPD patients. Data were pooled from eleven randomized, doubleblind clinical studies: a 52-week study of indacaterol $300 \mu \mathrm{g}$ and $600 \mu \mathrm{g}$, formoterol $12 \mu \mathrm{g}$, and placebo; ${ }^{3}$ a 26 -week study of indacaterol $150 \mu \mathrm{g}$ and $300 \mu \mathrm{g}$, placebo, and open-label tiotropium $18 \mu \mathrm{g}^{4}$ and an extension of this study providing data on 52 weeks of treatment with indacaterol $150 \mu \mathrm{g}$ and $300 \mu \mathrm{g}$ and placebo; ${ }^{6}$ a 26-week comparison of indacaterol $150 \mu \mathrm{g}$, salmeterol $50 \mu \mathrm{g}$, and placebo; ${ }^{5}$ a 12-week comparison of indacaterol $150 \mu \mathrm{g}$ and placebo; ${ }^{2}$ two 12 -week studies comparing indacaterol $150 \mu \mathrm{g}$ with salmeterol ${ }^{10}$ or tiotropium; ${ }^{11}$ two 12-week studies comparing indacaterol $75 \mu \mathrm{g}$ with placebo; ${ }^{12}$ and two pan-Asian studies of 12 and 26 weeks, comparing indacaterol $150 \mu \mathrm{g}$ and $300 \mu \mathrm{g}$ and placebo $^{13}$ (one unpublished; see ClinicalTrials.gov identifier NCT00792805). Data from additional treatment arms (indacaterol $75 \mu \mathrm{g}$ and $600 \mu \mathrm{g}$ and formoterol $12 \mu \mathrm{g}$ twice daily) in an initial dose-finding stage of one study are also included. ${ }^{14}$ Indacaterol and tiotropium were taken once daily, salmeterol and formoterol twice daily. Further details of the eleven studies are provided in Table S1. A further analysis was undertaken specifically to look for any effect of treatment on acute respiratory serious adverse events. For this purpose, data were analyzed from all blinded studies (23 in total) in COPD patients of at least 7 days' duration in which indacaterol was taken via single-dose dry powder inhaler; only data from blinded treatments were included.

\section{Assessments and outcomes}

All the studies collected information on adverse events, serious adverse events, and deaths (a subset of serious adverse events). An adverse event was defined as the appearance or worsening of any undesirable sign, symptom, or medical condition occurring after starting the study. Adverse events were categorized using the Medical Dictionary for Regulatory Activities (MedDRA), the clinically validated, international medical terminology used by regulatory authorities and the pharmaceutical industry in the USA and EU. MedDRA is organized in different levels, from "system organ class" down to "preferred terms" for individual adverse events. Because of the overlap between related preferred terms, the terms may also be arranged in groups of terms relevant to a defined medical condition or area of interest, known as standardized MedDRA query groups.

The preferred term "COPD" is described here as "COPD worsening." Although this term captured any exacerbations, it also included events of any severity described as "worsening of COPD" and thus lacks sufficient rigor to merit the description "exacerbations." Several of the studies in this safety evaluation did analyze COPD exacerbations that were defined and recorded separately from "COPD worsening" as an adverse event. For this analysis, a "COPD exacerbation" was defined as the onset or worsening of more than one respiratory symptom (ie, dyspnea, cough, sputum purulence or volume, or wheeze) for more than 3 consecutive days, plus a documented change in COPD-related treatment due to worsening symptoms (eg, corticosteroids, antibiotics, oxygen), and/or a documented COPD-related hospitalization or emergency room visit. In later studies, ${ }^{10-12,15}$ the definition was updated to a worsening of two or more major symptoms (dyspnea, sputum volume and sputum purulence) for $\geq 2$ consecutive days that required treatment with corticosteroids and/or antibiotics, or a worsening of any one of those symptoms plus any one minor symptom (sore throat, colds, fever without other cause, increased cough, increased wheeze) for $\geq 2$ consecutive days that required treatment with corticosteroids and/or antibiotics. To better understand the profile of cardiovascular safety, additional analyses were performed using a predefined search for "major adverse cardiovascular events" (MACE). The MACE search was devised by the Division of Metabolism and Endocrinology Products at the US Food and Drug Administration (FDA) to analyze potential cardiovascular and cerebrovascular risks for new therapies. ${ }^{16}$ There are two MACE searches: (1) a broad search including all preferred terms relating to myocardial infarction, cerebrovascular events, and nervous system hemorrhages; and (2) a custom MACE (a subset of broad MACE), in which the search terms were selected by FDA medical reviewers to best match those referring to myocardial infarction and stroke. (For the terms used, see Tables S3 and S4 in the Supplementary material.) All fatal 
events were adjudicated independently in a blinded fashion to determine which were primarily cardiac in nature, and these were included in both broad and custom MACE searches.

A "serious adverse event" was defined as an event that: was fatal or life threatening, resulted in persistent or significant disability/incapacity, constituted a congenital anomaly/ birth defect, required inpatient hospitalization or prolongation of existing hospitalization (with certain exceptions), or was medically significant (an event that jeopardized the patient or required medical or surgical intervention to prevent one of the outcomes listed above). All deaths occurring after intake of the first dose of the study drug up to and including 30 days after the patient's last dose of study drug were included in the present analysis.

An additional analysis of serious adverse events was conducted using a composite endpoint of acute respiratory events resulting in hospitalization, intubation, or death. ${ }^{17}$ Data for all serious adverse events occurring in patients in studies of at least 7 days in length (including studies in COPD and asthma) were supplied to an adjudication committee of five pulmonologists who were independent of the manufacturers of indacaterol. The committee, which was blinded to treatment, reviewed details of each event to determine if it was due to an acute worsening of underlying COPD or asthma or to pneumonia. Results of the 23 studies conducted in COPD patients are reported here.

Since systemic $\beta_{2}$-adrenoceptor stimulation may cause tachycardia and various metabolic effects, ${ }^{18}$ variables that reflect the effect of treatment on systemic $\beta_{2}$-adrenoceptors are also presented, including plasma potassium, blood glucose, and the electrocardiogram (ECG)-derived QTc interval. Measurements of vital signs (pulse and blood pressure), ECG, plasma potassium, and blood glucose levels were made at baseline and at intervals during the treatment periods.

Early in the indacaterol clinical development program, it was noticed that some patients had a transient cough shortly after indacaterol inhalation. Later, and in the studies included here, this cough was monitored and its time to onset and duration recorded separately by investigators. The incidence of cough was also evaluated for possible associations, for example with bronchospasm or treatment discontinuation.

\section{Patients}

The studies had almost identical patient entry criteria. Patients were to be at least 40 years of age at screening, with a smoking history of 10-20 pack-years (depending on study), and a clinical diagnosis of moderate-to-severe COPD defined according to Global Initiative for Chronic Obstructive Lung Disease (GOLD) guidelines in 2005 or 2007; ie, post-bronchodilator forced expiratory volume in 1 second $\left(\mathrm{FEV}_{1}\right)<80 \%$ and $\geq 30 \%$ of the predicted normal value, and post-bronchodilator $\mathrm{FEV}_{1} /$ forced vital capacity $<70 \%$ (post-bronchodilator referring to $10-15$ or 30 minutes after inhalation of salbutamol $400 \mu \mathrm{g}$ ).

\section{Statistical analysis}

Given the different lengths of treatment in these studies, the pooled data from this patient population were presented in two ways. First, analyses of all data from all studies were used for adverse events, exacerbations, and deaths, with results adjusted for length of exposure and reported as incidence (number of events per patient-year of treatment). The indacaterol $150 \mu \mathrm{g}$ dose was best represented in terms of patient numbers and treatment duration, and it was used as the index treatment group for determining the order of incidence of "common" adverse events. Second, data on the "acute" effects of treatment (pulse, blood pressure, the ECGderived QTc interval, plasma potassium, and blood glucose) were analyzed for the first 3 months of treatment (including any post-baseline measurement) and were not adjusted for exposure. The 3-month data were also used for the analysis of adverse events by severity. One advantage of analyzing these data over the 3-month period (and one reason why adjustment for exposure is not required) is that the studies included had a minimum duration of 3 months. In addition, since these acute effects are determined from data collected at discrete time points (in contrast to adverse events, which could occur at any time during the study), the authors believe that it is not valid to correct these data for exposure.

Risk ratios for adverse events (active versus placebo) and $95 \%$ confidence intervals (CI) were obtained by Poisson regression with treatment and study in the model as class effects. The relative risk of death (active versus placebo) and $95 \% \mathrm{CI}$ were calculated through Poisson regression with treatment as a factor in the model. For the MACE analysis, the hazard ratios versus placebo were obtained from a Cox proportional hazards regression model stratified by study and country with treatment, inhaled corticosteroid (ICS) use, study, and seven baseline cardiovascular risk factors included as covariates. (Cardiovascular risk factors were: baseline age $\geq 65$ years, current smoker, cardio- or cerebrovascular condition, hypertension, hyperlipidemia, diabetes mellitus, and body mass index $>30 \mathrm{~kg} / \mathrm{m}^{2}$.) Hazard ratios for time to first acute respiratory serious adverse event were 
obtained from a Cox regression model stratified by country and study, with treatment, age, baseline $\mathrm{FEV}_{1}$, and albuterol reversibility as factors and the interaction of treatment and age as covariates. The results for the indacaterol (all) group for risk of MACE event and death were based on a weighted (by size) average of the indacaterol doses.

The rate of COPD exacerbations was analyzed for the intent-to-treat population of patients, including all randomized patients who received at least one dose of study drug, analyzed according to the treatment to which they were randomized. The analysis used a negative binomial model including terms for treatment, smoking status, ICS use, baseline COPD exacerbation history, baseline $\mathrm{FEV}_{1}$ reversibility components, study, and country. As exacerbation rate was an efficacy endpoint, the above-therapeutic indacaterol $600 \mu \mathrm{g}$ dose was not included in this analysis. All these methods take account of the varying exposure lengths across the studies.

Results for sitting pulse, sitting blood pressure, QTc interval, plasma potassium, and blood glucose are presented as percentages of patients with notable values and as least squares means and associated 95\% CI.

\section{Results}

\section{Patients and exposure to treatment}

Table 1 shows the length of time for which patients were exposed to study drugs. The $75 \mu \mathrm{g}$ dose of indacaterol was only evaluated in studies of 12 weeks' duration. Patients in all treatment groups had a mean age of 63-64 years and a mean duration of COPD of 7 years. $\mathrm{FEV}_{1} \%$ predicted was $53 \%-55 \%$ across the treatment groups. Other baseline characteristics of the patients are summarized in Table 2 . Treatment groups were reasonably well matched apart from ethnic origin, notably the proportion of Asian patients. Of note, approximately $40 \%$ of patients had three or more pre-existing cardiovascular risk factors and approximately $20 \%$ had a concomitant cardio- or cerebrovascular condition. Completion rates and reasons for withdrawal from the treatment groups are shown in Table S2.

\section{Adverse events}

Table 3 shows the overall adverse events and the most common adverse events ( $\geq 0.05$ incidence in the indacaterol $150 \mu$ g group), arranged within their respective system organ classes. The five most common events with indacaterol were COPD worsening, nasopharyngitis, headache, cough and upper respiratory tract infection (URTI). COPD worsening was the most common event in all treatment groups. The incidence of COPD worsening with all active treatments was lower than with placebo, and the upper $95 \%$ of the CIs for the associated relative risks were all $<1$, indicating a significant reduction compared with placebo. For the other most common adverse events, there were no consistent increases or decreases in incidence with indacaterol compared with placebo, and no relationship to indacaterol dose. For muscle spasms, the sixth most common adverse event, the incidence was increased relative to placebo with indacaterol 150, 300, and $600 \mu \mathrm{g}$, although, again, this did not appear to be related to dose.

Severity of adverse events was analyzed up to the 3-month time point. Those events occurring most commonly with indacaterol were generally mild or moderate in severity, with a minority recorded as severe: COPD worsening, $9.0 \%$ of cases; nasopharyngitis, $1.6 \%$; headache, $4.8 \%$; cough, $1.8 \%$; URTI, $1.5 \%$, and muscle spasms, $9.9 \%$.

Besides headache and muscle spasms (Table 3), the incidence of other adverse events commonly associated with $\beta_{2}$-agonist class effects, including insomnia, anxiety, tremor, palpitations, and tachycardia, are presented in Table 4. There

Table I Duration of exposure to study drug after randomization

\begin{tabular}{|c|c|c|c|c|c|c|c|c|}
\hline & $\begin{array}{l}\text { IND } 75 \\
n=449\end{array}$ & $\begin{array}{l}\text { IND I50 } \\
n=26 \text { I I }\end{array}$ & $\begin{array}{l}\text { IND } 300 \\
\mathrm{n}=\mathrm{I} \text { I } 57\end{array}$ & $\begin{array}{l}\text { IND } 600 \\
n=547\end{array}$ & $\begin{array}{l}\text { FOR } \\
n=556\end{array}$ & $\begin{array}{l}\text { SLM } \\
\mathrm{n}=\mathbf{8 9 5}\end{array}$ & $\begin{array}{l}\text { TIO } \\
n=12 \mid 4\end{array}$ & $\begin{array}{l}\text { PBO } \\
n=2012\end{array}$ \\
\hline Exposure, days, & 85.5 & 120.3 & 232.7 & 263.4 & 260.3 & 112.2 & 107.7 & 167.7 \\
\hline mean (SD) & $(25.8 I)$ & $(74.61)$ & $(123.27)$ & $(135.27)$ & $(133.46)$ & $(5 \mathrm{l} .45)$ & $(49.36)$ & $(I \mid 5.93)$ \\
\hline \multirow[t]{2}{*}{ Median (range) } & 85.0 & 85.0 & 183.0 & 364.0 & 363.0 & 85.0 & 85.0 & 176.0 \\
\hline & $(2-179)$ & $(I-385)$ & $(I-420)$ & $(I-407)$ & $(I-397)$ & $(I-2 \mid 5)$ & $(I-208)$ & $(I-403)$ \\
\hline Patient-years & 105.1 & 859.7 & 737.0 & 394.5 & 396.2 & 274.9 & 358.0 & 923.6 \\
\hline \multicolumn{9}{|c|}{ Exposure, $\%$ of patients } \\
\hline$<\mathrm{I}$ mo & 4.5 & 4.6 & 6.2 & 5.7 & 5.2 & 4.9 & 5.1 & 9.1 \\
\hline $\mathrm{I}-<3 \mathrm{mo}$ & 75.5 & 60.3 & 13.7 & 13.0 & 12.2 & 60.1 & 61.9 & 34.7 \\
\hline $3-<6 \mathrm{mo}$ & 20.0 & 9.8 & II.4 & 15.9 & 18.2 & 9.3 & 13.3 & 10.1 \\
\hline $6-<12 \mathrm{mo}$ & 0 & 22.3 & 46.6 & 34.9 & 34.2 & 25.7 & 19.8 & 34.2 \\
\hline$\geq 12 \mathrm{mo}$ & 0 & 3.0 & 22.1 & 30.5 & 30.2 & 0 & 0 & 11.9 \\
\hline
\end{tabular}

Abbreviations: IND, indacaterol; FOR, formoterol; SLM, salmeterol; TIO, tiotropium; PBO, placebo; SD, standard deviation; mo, month(s); n, number. 
Table 2 Patients' baseline and demographic characteristics and coexisting cardiovascular risk factors ${ }^{\mathrm{a}}$

\begin{tabular}{|c|c|c|c|c|c|c|c|c|}
\hline & $\begin{array}{l}\text { IND } 75 \\
n=449\end{array}$ & $\begin{array}{l}\text { IND I50 } \\
n=2611\end{array}$ & $\begin{array}{l}\text { IND } 300 \\
n=1 / 57\end{array}$ & $\begin{array}{l}\text { IND } 600 \\
\mathrm{n}=\mathbf{5 4 7}\end{array}$ & $\begin{array}{l}\text { FOR } \\
n=556\end{array}$ & $\begin{array}{l}\text { SLM } \\
n=895\end{array}$ & $\begin{array}{l}\text { TIO } \\
n=|2| 4\end{array}$ & $\begin{array}{l}\text { PBO } \\
n=2012\end{array}$ \\
\hline \multicolumn{9}{|l|}{ Age (\%) } \\
\hline$<65$ years & 53.2 & 52.5 & 49.6 & 53.4 & 50.7 & 53.7 & 54.0 & 52.3 \\
\hline $65-<75$ years & 34.1 & 36.0 & 37.5 & 36.4 & 36.9 & 35.4 & 35.8 & 36.8 \\
\hline$\geq 75$ years & 12.7 & 11.5 & 12.9 & 10.2 & 12.4 & 10.8 & 10.3 & 10.9 \\
\hline Males (\%) & 55.2 & 69.5 & 78.5 & 73.3 & 75.2 & 74.2 & 66.5 & 71.4 \\
\hline \multicolumn{9}{|l|}{ Race (\%) } \\
\hline Caucasian & 92.2 & 77.6 & 66.7 & 92.0 & 91.9 & 82.2 & 91.4 & 74.7 \\
\hline Black & 4.2 & 2.1 & I.I & I.I & 0.5 & 1.2 & 1.5 & 1.9 \\
\hline Asian & 2.5 & 17.7 & 30.1 & 2.7 & 2.3 & 13.4 & 4.3 & 20.6 \\
\hline Other & 1.1 & 2.6 & 2.1 & 4.2 & 5.2 & 3.1 & 2.8 & 2.8 \\
\hline \multicolumn{9}{|l|}{ Severity of COPD (\%) } \\
\hline GOLD stage II or less & 63.0 & 58.7 & 55.7 & 55.8 & 55.6 & 55.0 & 59.9 & 55.2 \\
\hline GOLD stage III or worse & 37.0 & 41.3 & 44.3 & 44.1 & 43.9 & 45.0 & 40.1 & 44.7 \\
\hline ICS use (current) (\%) & 41.7 & 43.6 & 42.4 & 48.8 & 50.4 & 45.9 & 48.5 & 41.0 \\
\hline Ex/current smoker (\%) & $52.1 / 47.9$ & $56.5 / 43.5$ & $60.9 / 39.5$ & $58.7 / 41.3$ & $59.2 / 40.8$ & $55.2 / 44.8$ & $55.7 / 44.3$ & $57.1 / 42.9$ \\
\hline Pack-years (mean [SD]) & $53.9(26.82)$ & $44.5(22.69)$ & $48.4(33.2 I)$ & $53.0(60.43)$ & $49.8(54.60)$ & $43.2(21.88)$ & $44.6(22.09)$ & $49.0(42.03)$ \\
\hline \multicolumn{9}{|l|}{ No. of CV risk factors ${ }^{\mathrm{a}}(\%)$} \\
\hline 0 & 3.3 & 7.1 & 9.0 & 6.8 & 7.9 & 6.7 & 6.7 & 7.2 \\
\hline 1 & 17.4 & 23.2 & 27.4 & 25.6 & 23.2 & 26.9 & 21.6 & 26.6 \\
\hline 2 & 31.0 & 28.2 & 28.4 & 28.0 & 28.4 & 25.3 & 29.4 & 26.4 \\
\hline 3 or more & 48.3 & 41.5 & 35.2 & 39.7 & 40.5 & $4 I .1$ & 42.3 & 39.8 \\
\hline Concomitant CCV condition & 22.5 & 18.2 & 16.9 & 20.8 & 20.5 & 20.2 & 19.9 & 19.0 \\
\hline
\end{tabular}

Note: ${ }^{\mathrm{C}} \mathrm{CV}$ risk factors were: age $\geq 65$, current smoker, $\mathrm{CCV}$ condition, hypertension, hyperlipidemia, diabetes mellitus, and body mass index $>30 \mathrm{~kg} / \mathrm{m}^{2}$ at baseline. Abbreviations: IND, indacaterol; FOR, formoterol; SLM, salmeterol; TIO, tiotropium; PBO, placebo; COPD, chronic obstructive pulmonary disease; GOLD, Global Initiative for Chronic Obstructive Lung Disease; ICS, inhaled corticosteroids; CV, cardiovascular; CCV, cardio- or cerebrovascular; n, number.

were no consistent patterns of significant changes caused by active compared with placebo treatment. The only events recorded as severe (from the 3-month data) for indacaterol doses combined were one event each of anxiety, palpitations, and tachycardia. None of the insomnia or tremor events was considered severe.

\section{Major adverse cardiovascular events (MACE) analysis}

Results of the MACE analysis are shown in Table 5. For both the broad and the more focused custom MACE categories, incidence was numerically lower with all active treatments than with placebo. Hazard ratios versus placebo (Figure 1) showed a nonsignificant reduction with all active treatments apart from tiotropium, which was nonsignificantly increased. There was no relationship with indacaterol dose.

\section{Deaths and serious adverse events}

The number of deaths adjusted per patient-year (Table 6) was lower with all indacaterol dose groups and with the other active treatments, than with placebo, and there was no relationship with indacaterol dose. Fourteen deaths occurred in the placebo group, with causes listed as: COPD exacerbation (1), cardiac arrest (1), cardiorespiratory arrest (1), myocardial infarction or possible myocardial infarction (3), sudden death (3), ruptured aortic aneurysm (2), multiorgan failure (1), accidental fall (1), and unknown (1). There were four deaths in the indacaterol $150 \mu \mathrm{g}$ group, none of which was suspected of being treatment related (causes were: sudden death, cardiac arrest and death, cardiopulmonary failure and gastric cancer, and acute myocardial infarction). There were two deaths in the indacaterol $300 \mu \mathrm{g}$ group (one due to cardiac arrest, one due to myocardial infarction), after 87 and 232 days of treatment, both of which were suspected to be treatment related. One death due to a COPD exacerbation after 189 days of indacaterol $600 \mu \mathrm{g}$ treatment was also suspected to be treatment related. The relative risk of death compared with placebo (Figure 2) was reduced with LABA treatment, with $P$-values that were significant (all indacaterol doses combined, $P=0.008$; salmeterol, $P=0.045$ ) or close to significant (indacaterol $300 \mu \mathrm{g}, P=0.054$; indacaterol $150 \mu \mathrm{g}, P=0.068$ ), excluding the $75 \mu \mathrm{g}$ group in which no deaths occurred. Relative risks versus placebo for formoterol and tiotropium were slightly higher than those with the other active treatments.

The most commonly occurring serious adverse events are listed in Table 6 . The overall incidence of serious adverse events was similar (and numerically lower) with 
Table 3 Incidence (events per patient-year) of most common adverse events (includes all events with $\geq 0.05$ incidence in any indacaterol group), adjusted for length of time on treatment, sorted by (a) primary system organ class and (b) preferred term, with relative risks versus placebo and $95 \% \mathrm{Cl}$

\begin{tabular}{|c|c|c|c|c|c|c|c|c|}
\hline & $\begin{array}{l}\text { IND } 75 \\
n=449\end{array}$ & $\begin{array}{l}\text { IND I50 } \\
n=26 I I\end{array}$ & $\begin{array}{l}\text { IND } 300 \\
n=1157\end{array}$ & $\begin{array}{l}\text { IND } 600 \\
n=547\end{array}$ & $\begin{array}{l}\text { FOR } \\
n=556\end{array}$ & $\begin{array}{l}\text { SLM } \\
n=895\end{array}$ & $\begin{array}{l}\text { TIO } \\
n=12 \mid 4\end{array}$ & $\begin{array}{l}\text { PBO } \\
n=2012\end{array}$ \\
\hline Total patient years & 105.06 & 859.72 & 736.97 & 394.49 & 396.21 & 274.93 & 357.97 & 923.60 \\
\hline AE episodes/patient-year & 5.39 & 4.10 & 3.92 & 3.56 & 2.95 & 3.02 & 4.45 & 3.88 \\
\hline $\begin{array}{l}\text { Respiratory, thoracic and } \\
\text { mediastinal disorders (total) }\end{array}$ & 1.26 & 0.93 & 1.05 & 0.88 & 0.80 & 0.69 & 1.10 & 1.07 \\
\hline COPD worsening & 0.41 & 0.46 & 0.55 & 0.54 & 0.59 & 0.35 & 0.48 & 0.67 \\
\hline Relative risk $(95 \% \mathrm{Cl})$ & $\begin{array}{l}0.66 \\
(0.47,0.93)\end{array}$ & $\begin{array}{l}0.76 \\
(0.66,0.88)\end{array}$ & $\begin{array}{l}0.80 \\
(0.70,0.91)\end{array}$ & $\begin{array}{l}0.74 \\
(0.63,0.88)\end{array}$ & $\begin{array}{l}0.75 \\
(0.64,0.89)\end{array}$ & $\begin{array}{l}0.71 \\
(0.54,0.92)\end{array}$ & $\begin{array}{l}0.64 \\
(0.52,0.79)\end{array}$ & \\
\hline - in patients on ICS & 0.54 & 0.64 & 0.74 & 0.68 & 0.64 & 0.48 & 0.63 & 0.79 \\
\hline - in patients not on ICS & 0.32 & 0.33 & 0.40 & 0.40 & 0.46 & 0.24 & 0.35 & 0.58 \\
\hline Cough & 0.30 & 0.17 & 0.16 & 0.12 & 0.07 & 0.08 & 0.16 & 0.12 \\
\hline Relative risk $(95 \% \mathrm{Cl})$ & $\begin{array}{l}1.38 \\
(0.86,2.22)\end{array}$ & $\begin{array}{l}\text { I. } 17 \\
(0.88,1.55)\end{array}$ & $\begin{array}{l}1.52 \\
(1.15,2.00)\end{array}$ & $\begin{array}{l}1.29 \\
(0.88,1.89)\end{array}$ & $\begin{array}{l}0.75 \\
(0.48, I .18)\end{array}$ & $\begin{array}{l}0.87 \\
(0.49,1.52)\end{array}$ & $\begin{array}{l}0.83 \\
0.57,1.21 \text { ) }\end{array}$ & \\
\hline Dyspnea & 0.10 & 0.06 & 0.05 & 0.06 & 0.04 & 0.06 & 0.08 & 0.08 \\
\hline Relative risk $(95 \% \mathrm{Cl})$ & $\begin{array}{l}0.72 \\
(0.34,1.52)\end{array}$ & $\begin{array}{l}0.60 \\
(0.40,0.91)\end{array}$ & $\begin{array}{l}0.68 \\
(0.45,1.04)\end{array}$ & $\begin{array}{l}0.98 \\
(0.59,1.62)\end{array}$ & $\begin{array}{l}0.58 \\
(0.32,1.06)\end{array}$ & $\begin{array}{l}0.52 \\
(0.27,1.00)\end{array}$ & $\begin{array}{l}0.59 \\
(0.35,1.01)\end{array}$ & \\
\hline Oropharyngeal pain & 0.11 & 0.05 & 0.05 & 0.02 & 0.02 & 0.04 & 0.08 & 0.03 \\
\hline Relative risk $(95 \% \mathrm{Cl})$ & $\begin{array}{l}2.96 \\
(1.18,7.43)\end{array}$ & $\begin{array}{l}1.86 \\
(1.07,3.22)\end{array}$ & $\begin{array}{l}1.60 \\
(0.93,2.75)\end{array}$ & $\begin{array}{l}1.09 \\
(0.48,2.5 I)\end{array}$ & $\begin{array}{l}0.84 \\
(0.34,2.08)\end{array}$ & $\begin{array}{l}1.98 \\
(0.74,5.30)\end{array}$ & $\begin{array}{l}2.47 \\
(1.27,4.83)\end{array}$ & \\
\hline Infections and infestations (total) & 1.25 & 0.94 & 1.07 & 0.97 & 0.77 & 0.66 & 1.13 & 0.96 \\
\hline Nasopharyngitis & 0.27 & 0.22 & 0.27 & 0.32 & 0.22 & 0.19 & 0.24 & 0.22 \\
\hline Relative risk $(95 \% \mathrm{Cl})$ & $\begin{array}{l}1.52 \\
(0.94,2.46)\end{array}$ & $\begin{array}{l}1.21 \\
(0.96,1.5 \mathrm{I})\end{array}$ & $\begin{array}{l}1.20 \\
(0.98,1.47)\end{array}$ & $\begin{array}{l}1.28 \\
(1.01,1.64)\end{array}$ & $\begin{array}{l}0.89 \\
(0.68,1.17)\end{array}$ & $\begin{array}{l}1.26 \\
(0.86,1.85)\end{array}$ & $\begin{array}{l}1.14 \\
(0.84,1.55)\end{array}$ & \\
\hline Upper RTI (5) & 0.17 & 0.11 & 0.13 & 0.09 & 0.07 & 0.03 & 0.14 & 0.12 \\
\hline Relative risk $(95 \% \mathrm{Cl})$ & $\begin{array}{l}1.05 \\
(0.59,1.85)\end{array}$ & $\begin{array}{l}0.91 \\
(0.68,1.23)\end{array}$ & $\begin{array}{l}0.96 \\
(0.72,1.27)\end{array}$ & $\begin{array}{l}1.07 \\
(0.71,1.62)\end{array}$ & $\begin{array}{l}0.85 \\
(0.55,1.33)\end{array}$ & $\begin{array}{l}0.46 \\
(0.19,1.12)\end{array}$ & $\begin{array}{l}0.95 \\
(0.64,1.39)\end{array}$ & \\
\hline Upper RTI bacterial & 0.06 & 0.06 & 0.09 & 0.09 & 0.08 & 0.02 & 0.05 & 0.09 \\
\hline Relative risk $(95 \% \mathrm{Cl})$ & $\begin{array}{l}0.65 \\
(0.25,1.67)\end{array}$ & $\begin{array}{l}0.77 \\
(0.50,1.18)\end{array}$ & $\begin{array}{l}0.89 \\
(0.64,1.24)\end{array}$ & $\begin{array}{l}0.71 \\
(0.47,1.07)\end{array}$ & $\begin{array}{l}0.60 \\
(0.39,0.94)\end{array}$ & $\begin{array}{l}0.19 \\
(0.06,0.57)\end{array}$ & $\begin{array}{l}0.53 \\
(0.29,0.98)\end{array}$ & \\
\hline Lower RTI & 0.02 & 0.06 & 0.09 & 0.10 & 0.08 & 0.09 & 0.06 & 0.09 \\
\hline Relative risk $(95 \% \mathrm{Cl})$ & $\begin{array}{l}0.21 \\
(0.05,0.90)\end{array}$ & $\begin{array}{l}0.79 \\
(0.53,1.18)\end{array}$ & $\begin{array}{l}0.83 \\
(0.60,1.16)\end{array}$ & $\begin{array}{l}0.85 \\
(0.56,1.28)\end{array}$ & $\begin{array}{l}0.69 \\
(0.45,1.07)\end{array}$ & $\begin{array}{l}1.50 \\
(0.81,2.77)\end{array}$ & $\begin{array}{l}0.62 \\
(0.36,1.07)\end{array}$ & \\
\hline Influenza & 0.04 & 0.05 & 0.05 & 0.07 & 0.04 & 0.03 & 0.06 & 0.04 \\
\hline Relative risk $(95 \% \mathrm{Cl})$ & $\begin{array}{l}1.57 \\
(0.49,5.05)\end{array}$ & $\begin{array}{l}1.08 \\
(0.62,1.91)\end{array}$ & $\begin{array}{l}\text { I.3I } \\
(0.82,2.09)\end{array}$ & $\begin{array}{l}1.33 \\
(0.77,2.30)\end{array}$ & $\begin{array}{l}0.76 \\
(0.40,1.44)\end{array}$ & $\begin{array}{l}\text { I.II } \\
(0.43,2.90)\end{array}$ & $\begin{array}{l}0.70 \\
(0.36,1.40)\end{array}$ & \\
\hline Bronchitis & 0.12 & 0.05 & 0.06 & 0.06 & 0.04 & 0.03 & 0.06 & 0.07 \\
\hline Relative risk $(95 \% \mathrm{Cl})$ & $\begin{array}{l}1.06 \\
(0.54,2.12)\end{array}$ & $\begin{array}{l}0.75 \\
(0.48,1.17)\end{array}$ & $\begin{array}{l}0.97 \\
(0.65,1.45)\end{array}$ & $\begin{array}{l}0.91 \\
(0.54,1.54)\end{array}$ & $\begin{array}{l}0.69 \\
(0.39,1.23)\end{array}$ & $\begin{array}{l}1.18 \\
(0.42,3.39)\end{array}$ & $\begin{array}{l}0.52 \\
(0.30,0.91)\end{array}$ & \\
\hline Viral upper RTI & 0.05 & 0.05 & 0.06 & 0.03 & 0.04 & 0.02 & 0.04 & 0.05 \\
\hline Relative risk $(95 \% \mathrm{Cl})$ & $\begin{array}{l}0.84 \\
(0.29,2.4 I)\end{array}$ & $\begin{array}{l}1.21 \\
(0.76,1.94)\end{array}$ & $\begin{array}{l}1.34 \\
(0.86,2.11)\end{array}$ & $\begin{array}{l}0.57 \\
(0.28,1.18)\end{array}$ & $\begin{array}{l}0.85 \\
(0.45,1.60)\end{array}$ & $\begin{array}{l}0.41 \\
(0.15,1.14)\end{array}$ & $\begin{array}{l}0.67 \\
(0.33,1.35)\end{array}$ & \\
\hline Nervous system disorders (total) & 0.47 & 0.34 & 0.20 & 0.22 & 0.17 & 0.25 & 0.33 & 0.23 \\
\hline Headache & 0.33 & 0.18 & 0.07 & 0.11 & 0.08 & 0.14 & 0.18 & 0.10 \\
\hline Relative risk $(95 \% \mathrm{Cl})$ & $\begin{array}{l}2.34 \\
(1.46,3.76)\end{array}$ & $\begin{array}{l}1.11 \\
(0.80,1.53)\end{array}$ & $\begin{array}{l}0.67 \\
(0.47,0.95)\end{array}$ & $\begin{array}{l}1.09 \\
(0.73,1.63)\end{array}$ & $\begin{array}{l}0.77 \\
(0.50,1.204)\end{array}$ & $\begin{array}{l}0.78 \\
(0.47,1.29)\end{array}$ & $\begin{array}{l}0.90 \\
(0.61,1.32)\end{array}$ & \\
\hline $\begin{array}{l}\text { Musculoskeletal and connective } \\
\text { tissue disorders (total) }\end{array}$ & 0.41 & 0.37 & 0.31 & 0.32 & 0.25 & 0.26 & 0.33 & 0.28 \\
\hline Muscle spasms & 0.06 & 0.10 & 0.07 & 0.11 & 0.05 & 0.06 & 0.02 & 0.04 \\
\hline Relative risk $(95 \% \mathrm{Cl})$ & $\begin{array}{l}1.30 \\
(0.47,3.59)\end{array}$ & $\begin{array}{l}2.42 \\
(1.58,3.7 I)\end{array}$ & $\begin{array}{l}2.08 \\
(1.34,3.24)\end{array}$ & $\begin{array}{l}3.48 \\
(2.10,5.78)\end{array}$ & $\begin{array}{l}1.69 \\
(0.93,3.04)\end{array}$ & $\begin{array}{l}1.26 \\
(0.63,2.54)\end{array}$ & $\begin{array}{l}0.66 \\
(0.29,1.49)\end{array}$ & \\
\hline Arthralgia & 0.10 & 0.05 & 0.04 & 0.03 & 0.02 & 0.02 & 0.03 & 0.03 \\
\hline Relative risk $(95 \% \mathrm{Cl})$ & $\begin{array}{l}2.14 \\
(0.88,5.20)\end{array}$ & $\begin{array}{l}\mathrm{I} .40 \\
(0.79,2.47)\end{array}$ & $\begin{array}{l}1.32 \\
(0.76,2.30)\end{array}$ & $\begin{array}{l}1.32 \\
(0.64,2.74)\end{array}$ & $\begin{array}{l}0.7 \mid \\
(0.29, \mid .7 I)\end{array}$ & $\begin{array}{l}0.42 \\
(0.14,1.27)\end{array}$ & $\begin{array}{l}0.61 \\
(0.27,1.38)\end{array}$ & \\
\hline Back pain & 0.06 & 0.05 & 0.04 & 0.05 & 0.07 & 0.06 & 0.06 & 0.06 \\
\hline Relative risk $(95 \% \mathrm{Cl})$ & $\begin{array}{l}1.05 \\
(0.39,2.78)\end{array}$ & $\begin{array}{l}0.86 \\
(0.55,1.35)\end{array}$ & $\begin{array}{l}0.73 \\
(0.46,1.16)\end{array}$ & $\begin{array}{l}0.84 \\
(0.47, I .5 I)\end{array}$ & $\begin{array}{l}1.20 \\
(0.71,2.03)\end{array}$ & $\begin{array}{l}1.18 \\
(0.56,2.47)\end{array}$ & $\begin{array}{l}0.80 \\
(0.44,1.47)\end{array}$ & \\
\hline
\end{tabular}

Abbreviations: IND, indacaterol; FOR, formoterol; SLM, salmeterol; TIO, tiotropium; PBO, placebo; AE, adverse event; COPD, chronic obstructive pulmonary disease; $\mathrm{Cl}$, confidence interval; ICS, inhaled corticosteroids; RTI, respiratory tract infection; n, number. 
Table 4 Incidence (events per patient-year) of selected adverse events commonly associated with $\beta_{2}$-adrenoceptor mediated effects, adjusted for length of time on treatment

\begin{tabular}{|c|c|c|c|c|c|c|c|c|}
\hline & $\begin{array}{l}\text { IND } 75 \\
n=449\end{array}$ & $\begin{array}{l}\text { IND I50 } \\
n=2611\end{array}$ & $\begin{array}{l}\text { IND } 300 \\
n=1 / 57\end{array}$ & $\begin{array}{l}\text { IND } 600 \\
n=547\end{array}$ & $\begin{array}{l}\text { FOR } \\
n=556\end{array}$ & $\begin{array}{l}\text { SLM } \\
n=895\end{array}$ & $\begin{array}{l}\text { TIO } \\
n=|2| 4\end{array}$ & $\begin{array}{l}\text { PBO } \\
n=2012\end{array}$ \\
\hline Total patient years & 105.06 & 859.72 & 736.97 & 394.49 & 396.21 & 274.93 & 357.97 & 923.60 \\
\hline Insomnia & 0.02 & 0.02 & 0.01 & 0.02 & 0.02 & 0.05 & 0.02 & 0.03 \\
\hline Relative risk $(95 \% \mathrm{Cl})$ & $\begin{array}{l}0.60 \\
(0.13,2.82)\end{array}$ & $\begin{array}{l}0.47 \\
(0.24,0.94)\end{array}$ & $\begin{array}{l}0.28 \\
(0.12,0.65)\end{array}$ & $\begin{array}{l}1.14 \\
(0.42,3.11)\end{array}$ & $\begin{array}{l}1.68 \\
(0.69,4.07)\end{array}$ & $\begin{array}{l}1.86 \\
(0.57,6.01)\end{array}$ & $\begin{array}{l}0.49 \\
(0.19,1.24)\end{array}$ & \\
\hline Anxiety & 0.02 & 0.02 & 0.01 & 0.02 & 0.01 & 0.02 & 0.01 & 0.02 \\
\hline Relative risk $(95 \% \mathrm{Cl})$ & $\begin{array}{l}0.48 \\
(0.10,2.36)\end{array}$ & $\begin{array}{l}0.79 \\
(0.32,1.97)\end{array}$ & $\begin{array}{l}0.88 \\
(0.36,2.15)\end{array}$ & $\begin{array}{l}\text { I. } 46 \\
(0.50,4.26)\end{array}$ & $\begin{array}{l}0.72 \\
(0.19,2.72)\end{array}$ & $\begin{array}{l}0.71 \\
(0.16,3.14)\end{array}$ & $\begin{array}{l}0.19 \\
(0.04,0.92)\end{array}$ & \\
\hline Tremor $^{\mathrm{a}}$ & 0 & 0.01 & 0.01 & 0.03 & 0.02 & 0.01 & 0.01 & 0.01 \\
\hline Palpitations & 0.01 & 0.01 & 0.01 & 0.01 & 0 & 0.02 & 0.02 & 0.01 \\
\hline Relative risk $(95 \% \mathrm{Cl})$ & $\begin{array}{l}0.98 \\
(0.09,10.6)\end{array}$ & $\begin{array}{l}0.22 \\
(0.05,0.96)\end{array}$ & $\begin{array}{l}0.69 \\
(0.29,1.64)\end{array}$ & $\begin{array}{l}0.27 \\
(0.06,1.27)\end{array}$ & $\begin{array}{l}0.14 \\
(0.02,1.08)\end{array}$ & $\begin{array}{l}0.54 \\
(0.08,3.83)\end{array}$ & $\begin{array}{l}0.83 \\
(0.19,3.70)\end{array}$ & \\
\hline Tachycardia & 0.01 & 0 & 0.01 & 0.01 & 0.01 & 0.01 & 0.01 & 0.01 \\
\hline Relative risk $(95 \% \mathrm{Cl})$ & $\begin{array}{l}1.15 \\
(0.10,13.6)\end{array}$ & $\begin{array}{l}0.73 \\
(0.12,4.26)\end{array}$ & $\begin{array}{l}0.87 \\
(0.31,2.42)\end{array}$ & $\begin{array}{l}0.46 \\
(0.12,1.77)\end{array}$ & $\begin{array}{l}0.30 \\
(0.06,1.46)\end{array}$ & $\begin{array}{l}2.77 \\
(0.18,41.90)\end{array}$ & $\begin{array}{l}1.43 \\
(0.30,6.91)\end{array}$ & \\
\hline
\end{tabular}

Note: ${ }^{a}$ There are no risk ratios for tremor as the model did not converge and the output was unreliable.

Abbreviations: IND, indacaterol; FOR, formoterol; SLM, salmeterol; TIO, tiotropium; PBO, placebo; $\mathrm{Cl}$, confidence interval; n, number.

all indacaterol groups compared with placebo. The most common serious adverse event was COPD worsening, which was numerically lower with indacaterol (all doses) than placebo; as expected, given the definition of a serious adverse event, most of these episodes led to hospitalization. Comparing subgroups of patients receiving ICS or not at study entry, the incidence of COPD worsening as a serious adverse event was similar or lower in the non-ICS users compared with ICS users in the indacaterol $75 \mu \mathrm{g}$, $150 \mu \mathrm{g}$, and $300 \mu \mathrm{g}$ and salmeterol groups, and slightly higher in the non-ICS users compared with the ICS users with indacaterol $600 \mu \mathrm{g}$, formoterol, tiotropium, and placebo. Serious adverse events in the categories "infections and infestations" and "cardiac disorders" generally occurred at a similar incidence in active and placebo treatment groups, with no relationship between incidence and indacaterol dose.

Rates of acute respiratory serious adverse events (as adjudicated by the blinded independent committee) were not significantly increased with the bronchodilator treatments compared with placebo (Table 7). None of the active treatments was associated with a significant increase in risk relative to placebo.

\section{Rate of COPD exacerbations}

Rates of COPD exacerbations were significantly reduced relative to placebo with all the active treatments (Figure 3). There were no statistically significant differences between active treatments.

\section{Cough following indacaterol inhalation}

In the "all data" analysis, the mean percentage of attended visits at which patients experienced cough after inhalation of indacaterol ranged from $14.1 \%$ to $18.4 \%$ across the indacaterol dose groups, compared with $2 \%$ in the placebo group, increasing slightly with increasing indacaterol dose. For the majority of patients, the cough started within 15 seconds of inhalation and lasted for $\leq 15$ seconds; the median duration in the indacaterol groups at each visit was $\leq 6$ seconds. There

Table 5 Major adverse cardiovascular events (MACE) episodes

\begin{tabular}{|c|c|c|c|c|c|c|c|c|}
\hline & $\begin{array}{l}\text { IND } 75 \\
n=449\end{array}$ & $\begin{array}{l}\text { IND I50 } \\
n=26 I I\end{array}$ & $\begin{array}{l}\text { IND } 300 \\
n=|I| 57\end{array}$ & $\begin{array}{l}\text { IND } 600 \\
n=547\end{array}$ & $\begin{array}{l}\text { FOR } \\
n=556\end{array}$ & $\begin{array}{l}\text { SLM } \\
\mathrm{n}=\mathbf{8 9 5}\end{array}$ & $\begin{array}{l}\text { TIO } \\
n=12 \mid 4\end{array}$ & $\begin{array}{l}\text { PBO } \\
n=2012\end{array}$ \\
\hline Total patient years & 105.06 & 859.72 & 736.97 & 394.49 & 396.21 & 274.93 & 357.97 & 923.60 \\
\hline \multicolumn{9}{|l|}{ Broad MACE } \\
\hline Patients with $\geq$ I event, n (\%) & $4(0.89)$ & $21(0.80)$ & $8(0.69)$ & $6(1.10)$ & $7(1.26)$ & $7(0.78)$ & II (0.9I) & $29(1.44)$ \\
\hline Events per patient-year (n) & 0.04 & 0.04 & 0.01 & 0.02 & 0.02 & 0.03 & 0.04 & 0.04 \\
\hline \multicolumn{9}{|l|}{ Custom MACE } \\
\hline Patients with $\geq$ I event, n (\%) & $\mathrm{I}(0.22)$ & $14(0.54)$ & $5(0.43)$ & $3(0.55)$ & $4(0.72)$ & $4(0.45)$ & $4(0.33)$ & $19(0.94)$ \\
\hline Events per patient-year (n) & 0.01 & 0.02 & 0.01 & 0.01 & 0.01 & 0.02 & 0.01 & 0.02 \\
\hline
\end{tabular}

Abbreviations: IND, indacaterol; FOR, formoterol; SLM, salmeterol; TIO, tiotropium; PBO, placebo; n, number. 


\section{A. Broad MACE}

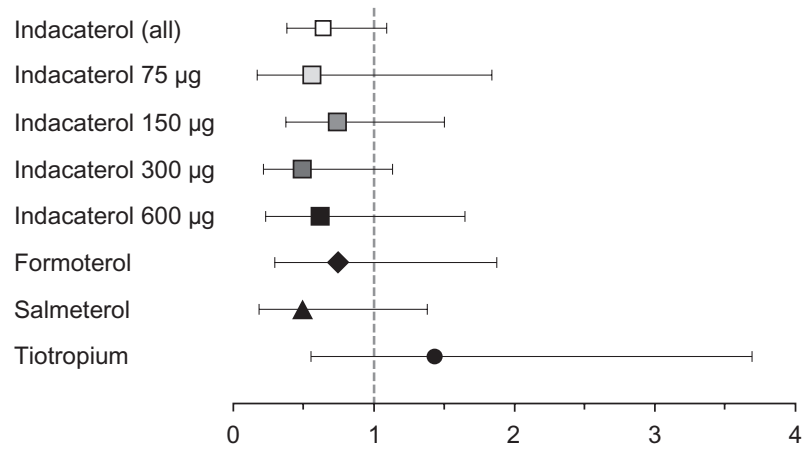

\section{B. Custom MACE}

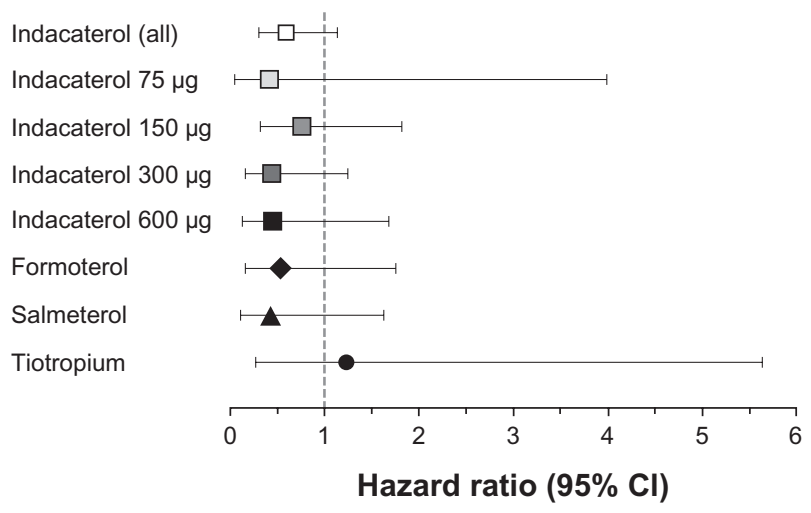

Figure I Risk of major adverse cardiovascular events (MACE) (myocardial infarction, stroke and cardiovascular death) relative to placebo, adjusted for length of time on treatment. (A) "Broad MACE" includes all preferred terms relating to myocardial infarction, cerebrovascular events, and nervous system hemorrhages; (B) "custom MACE" is a more focused subset of broad MACE including those preferred terms best describing myocardial infarction and stroke. (For the terms in each category, see Tables S3 and S4 in Supplementary material.)

Abbreviation: $\mathrm{Cl}$, confidence interval.

was no apparent association between the cough following indacaterol inhalation and decreased $\mathrm{FEV}_{1}$ or COPD exacerbations, or with reasons for or rates of withdrawal from the studies.

\section{$\beta_{2}$-adrenoceptor-mediated systemic effects}

Plasma potassium, blood glucose, and QTc interval values were assessed after 3 months' treatment (Table 8). The percentages of patients with notably low values of plasma potassium were not higher with any active treatment than with placebo, and there was no relationship between incidence of notable values and indacaterol dose. High blood glucose levels occurred at a similar rate in active and placebo treatment groups apart from a slightly higher rate in the indacaterol $600 \mu \mathrm{g}$ and salmeterol groups. The rate of high absolute values of QTc interval tended to be higher than placebo with most of the active treatment groups, but values $>500 \mathrm{~ms}$ were rare overall (reported in two patients each in the indacaterol $150 \mu \mathrm{g}$ and tiotropium groups). Increases from baseline in QTc interval of $>60 \mathrm{~ms}$ were less common among indacaterol-treated patients than those in the placebo group. Mean differences for these variables compared with placebo are shown in Figure 4 and, although CIs occasionally failed to cross zero, the differences were small and clinically insignificant.

\section{Vital signs}

The percentages of patients with notably fast or slow pulse and high or low values of blood pressure (Table 8) were generally similar between with active treatments and placebo. Small differences in mean values between active and placebo treatments and a trend to increasing pulse rate and decreasing blood pressure with increasing indacaterol dose were clinically insignificant (Figure 4).

\section{Discussion}

It is important to evaluate carefully the safety of any new drug for the long-term treatment of COPD, given that many patients are elderly and often have several comorbidities treated with multiple concomitant medications. ${ }^{19}$ The present study examines a relevant population of patients of whom about $50 \%$ were aged $>65$ years, with approximately $40 \%$ having three or more cardiovascular risk factors, including conditions such as hypertension and diabetes mellitus. The pooled patient population provided a substantial body of data and maximized exposure to study treatments, allowing a more robust comparison of treatment effects compared with single studies. The studies had very similar entry criteria, and outcomes were defined and evaluated in the same way across the studies.

Another important reason for examining the safety of a once-daily $\beta_{2}$-agonist bronchodilator is the history of safety concerns with the twice-daily $\beta_{2}$-agonists salmeterol and formoterol when used in asthma; specifically, the reported increased risk of asthma-related death and hospitalizations, which is generally thought to be mitigated by the concomitant use of ICS. ${ }^{20-22}$ While indacaterol is indicated only for use in COPD patients, the present data allow the examination of similar outcomes, for example, exacerbations of COPD and risks for death or hospitalization due to worsening of COPD. The risk of COPD worsening as an adverse event was significantly reduced with indacaterol compared with placebo. Similarly, the incidence of COPD worsening as a serious adverse event, including the subset of events leading to hospitalization, was numerically lower in all indacaterol treatment groups compared with placebo. There was also a general pattern for a lower 
Table 6 Deaths and serious (fatal and nonfatal) adverse events, with listing of most common serious adverse events (incidence $\geq 0.005$ per patient-year in indacaterol I50 $\mu \mathrm{g}$ group), adjusted for length of time on treatment

\begin{tabular}{|c|c|c|c|c|c|c|c|c|}
\hline & $\begin{array}{l}\text { IND } 75 \\
\mathrm{n}=449\end{array}$ & $\begin{array}{l}\text { IND I50 } \\
n=2611\end{array}$ & $\begin{array}{l}\text { IND } 300 \\
n=1157\end{array}$ & $\begin{array}{l}\text { IND } 600 \\
n=547\end{array}$ & $\begin{array}{l}\text { FOR } \\
n=556\end{array}$ & $\begin{array}{l}\text { SLM } \\
\mathrm{n}=895\end{array}$ & $\begin{array}{l}\text { TIO } \\
n=12 \mid 4\end{array}$ & $\begin{array}{l}\text { PBO } \\
n=2012\end{array}$ \\
\hline Total patient years & 105.06 & 859.72 & 736.97 & 394.49 & 396.21 & 274.93 & 357.97 & 923.60 \\
\hline Deaths (n) & 0 & 4 & 2 & 1 & 4 & 1 & 4 & 14 \\
\hline Deaths ( $\mathrm{n}$ per patient year) & 0 & 0.01 & 0 & 0 & 0.01 & 0 & 0.01 & 0.02 \\
\hline $\begin{array}{l}\text { Serious adverse events (fatal } \\
\text { and nonfatal) ( } \mathrm{n} \text { per patient year) }\end{array}$ & 0.25 & 0.24 & 0.24 & 0.21 & 0.32 & 0.18 & 0.33 & 0.27 \\
\hline $\begin{array}{l}\text { Respiratory, thoracic and } \\
\text { mediastinal disorders (total) }\end{array}$ & 0.07 & 0.05 & 0.09 & 0.04 & 0.12 & 0.06 & 0.06 & 0.09 \\
\hline COPD worsening & 0.05 & 0.05 & 0.06 & 0.03 & 0.10 & 0.04 & 0.04 & 0.07 \\
\hline - leading to hospitalization & 0.05 & 0.05 & 0.06 & 0.03 & 0.09 & 0.04 & 0.04 & 0.06 \\
\hline - in patients on ICS & 0.05 & 0.07 & 0.08 & 0.03 & 0.09 & 0.05 & 0.04 & 0.08 \\
\hline - in patients not on ICS & 0.05 & 0.03 & 0.04 & 0.04 & 0.11 & 0.03 & 0.04 & 0.05 \\
\hline Infections and infestations (total) & 0.05 & 0.04 & 0.04 & 0.02 & 0.08 & 0.04 & 0.05 & 0.05 \\
\hline Pneumonia & 0.02 & 0.01 & 0.01 & 0.01 & 0.02 & 0.01 & 0.02 & 0.01 \\
\hline Lower RTI & 0 & 0.01 & 0 & 0.01 & 0.01 & 0.01 & 0 & 0.01 \\
\hline URTI bacterial & 0.01 & 0.01 & 0 & 0 & 0.01 & 0.01 & 0 & 0.01 \\
\hline Cardiac disorders (total) & 0.04 & 0.03 & 0.03 & 0.03 & 0.02 & 0.03 & 0.05 & 0.03 \\
\hline Angina pectoris & 0.01 & 0.01 & 0 & 0 & 0 & 0 & 0 & 0 \\
\hline Acute myocardial infarction & 0 & 0.01 & 0 & 0 & 0 & 0 & 0 & 0 \\
\hline Atrial fibrillation & 0.01 & 0.01 & 0.01 & 0 & 0 & 0 & 0.01 & 0.01 \\
\hline
\end{tabular}

Abbreviations: IND, indacaterol; FOR, formoterol; SLM, salmeterol; TIO, tiotropium; PBO, placebo; COPD, chronic obstructive pulmonary disease; ICS, inhaled corticosteroids; RTI, respiratory tract infection; URTI, upper RTI; n, number.

incidence of COPD worsening as an adverse or serious adverse event in patients not receiving concomitant ICS (ie, who were receiving LABA monotherapy) compared with those who were receiving ICS. This is compatible with the GOLD guidelines that recommend patients with frequent exacerbations should be treated with ICS. ${ }^{1}$

The number of deaths during indacaterol treatment was extremely low, and the relative risk of all-cause death was reduced with indacaterol relative to placebo. These findings were borne out in the independently adjudicated analysis of all serious adverse events, which showed no

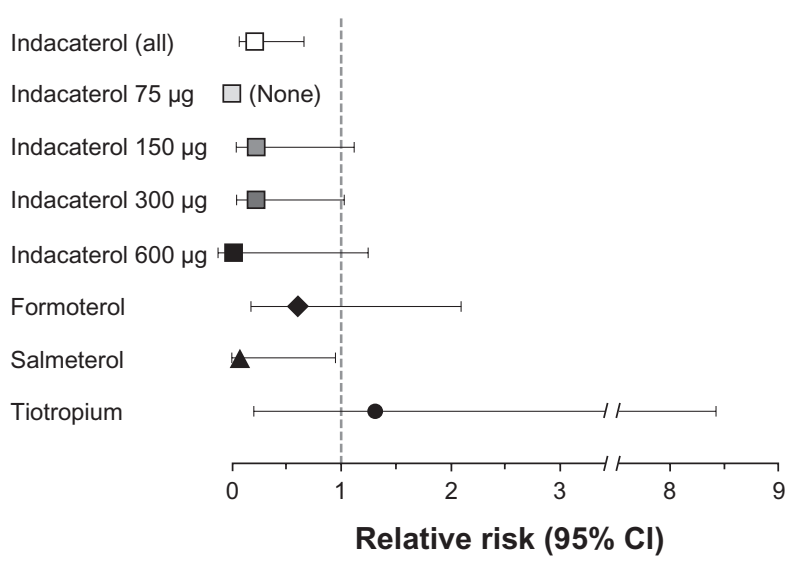

Figure 2 Risk of death relative to placebo, adjusted for length of time on treatment. Abbreviation: $\mathrm{Cl}$, confidence interval. significant increase in risk for the acute respiratory events leading to hospitalization, intubation, or death. Thus, LABA monotherapy with indacaterol in COPD was not associated with any increase in deaths, COPD worsening adverse events, serious adverse events, or hospitalizations. Indeed, the rate of COPD exacerbations, within the strict definition of this event when analyzed in the intent-to-treat population, was significantly reduced relative to placebo with indacaterol, the other LABAs, and with tiotropium.

Many of the common adverse events occurred with a similar incidence in the indacaterol and placebo groups and reflect the typical signs and symptoms of COPD itself. The majority of events, such as nasopharyngitis, cough, oropharyngeal pain, muscle spasms, arthralgia, and influenza, were mild or moderate in severity in all or a large majority of cases in each study. The incidence of muscle spasms was notably higher with the LABA treatments than with placebo, which may be related to stimulation of skeletal muscle $\beta_{2}$-adrenoceptors. While the analysis of tremor did not yield a risk ratio for statistical reasons, the risk ratios for other "typical" $\beta_{2}$-class effects of headache, insomnia, anxiety, palpitations, and tachycardia were not increased with indacaterol doses of 150 and $300 \mu \mathrm{g}$ compared with placebo, and were rarely severe. Results for QTc interval, plasma potassium, and blood glucose showed no clinically significant changes with indacaterol treatment. 
Table 7 Incidence (events per patient-year) of acute respiratory-related serious adverse events, ${ }^{a}$ adjusted for length of time on treatment

\begin{tabular}{|c|c|c|c|c|c|c|c|c|c|}
\hline & $\begin{array}{l}\text { IND } 75 \\
n=543\end{array}$ & $\begin{array}{l}\text { IND I } 50 \\
n=2745\end{array}$ & $\begin{array}{l}\text { IND } 300 \\
n=I 422\end{array}$ & $\begin{array}{l}\text { IND } 600 \\
n=584\end{array}$ & $\begin{array}{l}\text { IND DPIb }^{b} \\
n=6615\end{array}$ & $\begin{array}{l}\text { FOR } \\
n=556\end{array}$ & $\begin{array}{l}\text { SLM } \\
n=1010\end{array}$ & $\begin{array}{l}\text { TIO } \\
n=842\end{array}$ & $\begin{array}{l}\text { PBO } \\
n=2484\end{array}$ \\
\hline Total patient-years & 108.98 & 865.25 & 747.38 & 395.23 & 2381.78 & 396.21 & 279.64 & 179.39 & 940.98 \\
\hline $\begin{array}{l}\text { Acute respiratory- } \\
\text { related events }\end{array}$ & 0.06 & 0.05 & 0.07 & 0.04 & 0.06 & 0.10 & 0.04 & 0.04 & 0.06 \\
\hline COPD-related & 0.05 & 0.05 & 0.06 & 0.03 & 0.05 & 0.09 & 0.04 & 0.03 & 0.06 \\
\hline $\begin{array}{l}\text { Pneumonia- } \\
\text { related }\end{array}$ & 0.03 & 0.01 & 0.02 & 0.01 & 0.01 & 0.02 & 0.01 & 0.02 & 0.01 \\
\hline $\begin{array}{l}\text { Hazard ratio } \\
(95 \% \mathrm{Cl}) \text { vs placebo } \\
\text { for time to first } \\
\text { acute respiratory } \\
\text { event }\end{array}$ & $\begin{array}{l}\text { I.37 } \\
(0.49,3.78)\end{array}$ & $\begin{array}{l}0.84 \\
(0.51,1.40)\end{array}$ & $\begin{array}{l}1.05 \\
(0.70,1.59)\end{array}$ & $\begin{array}{l}0.61 \\
(0.33,1.13)\end{array}$ & $\begin{array}{l}0.93 \\
(0.69,1.24)\end{array}$ & $\begin{array}{l}\text { I. } 28 \\
(0.78,2.09)\end{array}$ & $\begin{array}{l}0.94 \\
(0.41,2.12)\end{array}$ & $\begin{array}{l}0.64 \\
(0.20,2.07)\end{array}$ & \\
\hline
\end{tabular}

Notes: Data from randomized, blinded treatment arms in studies of 7 days' duration or longer in patients with COPD; events were categorized by an independent adjudication committee who reviewed narratives for each event but who were blinded to treatment; adefined as events due to acute worsening of underlying condition and involving hospitalization (admission or emergency room visit $>24 \mathrm{~h}$ in duration), intubation (endotracheal intubation for mechanical ventilation for treatment of acute hypoxemic or hypercapneic respiratory failure) or death; acute events could be classified as related to COPD or pneumonia (as shown) or asthma (no events classified as such); ball patients receiving indacaterol via single-dose dry powder inhaler (includes 179 patients receiving indacaterol doses of 18.75 and $37.5 \mu g$ in dose-ranging studies).

Abbreviations: IND, indacaterol; FOR, formoterol; SLM, salmeterol; TIO, tiotropium; PBO, placebo; COPD, chronic obstructive pulmonary disease; CI, confidence interval; n, number.

In addition to the serious cases of COPD worsening discussed above, the incidence of serious adverse events was generally similar with active and placebo treatments, and again reflected typical disease manifestations in events such as pneumonia and respiratory tract infections. The overall incidence of serious adverse events in the category of cardiac disorders was similar between indacaterol and placebo.

Cardiovascular disorders were explored in more detail in the MACE analysis, in which the nonsignificant reductions in hazard ratio relative to placebo showed no indication of an association with indacaterol treatment for either the broad or more focused definition of cardiovascular events. Worth and colleagues recently published their analysis of the cardiovascular and cerebrovascular safety of indacaterol in a subset of the patients included in the current analysis, and also showed no potential for increased risks in these areas with indacaterol

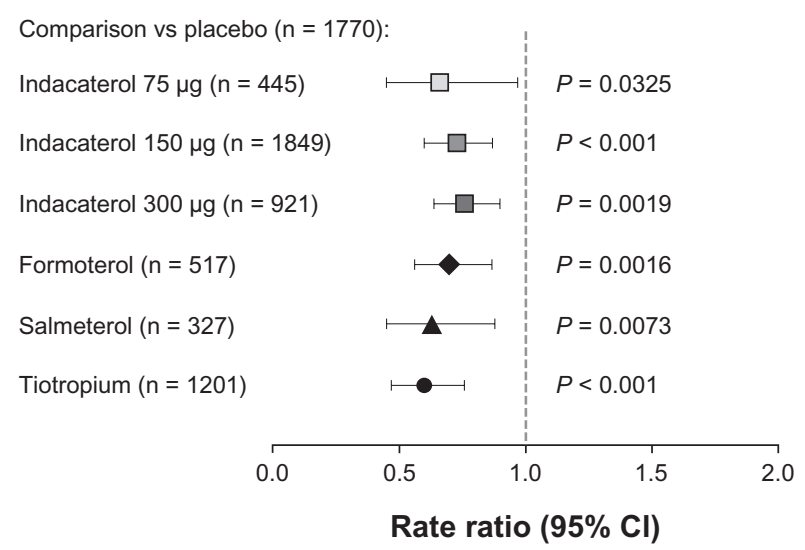

Figure 3 Rate of chronic obstructive pulmonary disease exacerbations compared with placebo over 12 months of treatment.

Abbreviation: $\mathrm{Cl}$, confidence interval. treatment. ${ }^{23}$ Similar findings were reported for salmeterol using the large 3-year Towards a Revolution in COPD Health (TORCH) study database, ${ }^{24}$ which found no increase in cardiovascular events (including serious and ischemic events and deaths) with salmeterol compared with placebo.

Few deaths occurred in any of the active treatment groups in the current analysis, and the number of deaths adjusted per patient-year was not increased with any of the LABA treatments relative to placebo. The finding of no deaths among the indacaterol $75 \mu \mathrm{g}$ group should be interpreted with caution, since most of the data came from two 12-week studies. A lack of association between LABA treatment and increased mortality in COPD patients has been reported by others. The TORCH study reported nonsignificant reductions with salmeterol in all-cause deaths (hazard ratio versus placebo 0.879 [95\% CI 0.729-1.061]) and in COPD-related deaths (hazard ratio versus placebo $1.01[95 \% \mathrm{CI} 0.76-1.35]) .{ }^{25}$ This finding was repeated in a meta-analysis of the available literature with LABAs in COPD, which also found a nonsignificant reduction in deaths with a LABA versus placebo (risk ratio $0.90[95 \%$ CI $0.77,1.06]) .{ }^{26} \mathrm{~A}$ large case-control study with more than 32,000 case patients found that LABA treatment was associated with significantly reduced odds of death (odds ratio 0.92 [95\% CI 0.88-0.96]) and a nonsignificant reduction in respiratory or cardiovascular deaths (odds ratio 0.98 [95\% CI 0.89-1.09]) compared with no treatment or with a short-acting $\beta_{2}$-agonist alone. ${ }^{27}$

There was no dose relationship with indacaterol in any of the categories of adverse events discussed above. Minor exceptions were seen with the cough following inhalation and with vital signs. However, the effects of indacaterol on 
Table 8 Percentages of patients with notable values for potassium (low) and glucose (high), blood pressures, pulse, and QTc interval

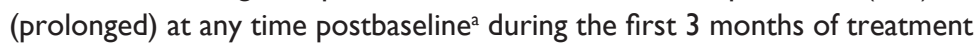

\begin{tabular}{|c|c|c|c|c|c|c|c|c|}
\hline & $\begin{array}{l}\text { IND } 75 \\
\mathrm{n}=449 \\
\end{array}$ & $\begin{array}{l}\text { IND I50 } \\
\mathrm{n}=\mathbf{2 6} \text { II }\end{array}$ & $\begin{array}{l}\text { IND } 300 \\
\mathrm{n}=11 \mathrm{I57}\end{array}$ & $\begin{array}{l}\text { IND } 600 \\
\mathrm{n}=\mathbf{5 4 7}\end{array}$ & $\begin{array}{l}\text { FOR } \\
\mathrm{n}=556\end{array}$ & $\begin{array}{l}\text { SLM } \\
\mathrm{n}=\mathbf{8 9 5}\end{array}$ & $\begin{array}{l}\text { TIO } \\
n=12 \mid 14\end{array}$ & $\begin{array}{l}\text { PBO } \\
n=2012\end{array}$ \\
\hline Potassium $<3.0 \mathrm{mmol} / \mathrm{L}$ & 0.2 & 0.1 & 0.2 & 0 & 0 & 0.2 & 0 & 0.2 \\
\hline Glucose $>9.99 \mathrm{mmol} / \mathrm{L}$ & 4.9 & 4.0 & 5.1 & 6.4 & 4.7 & 5.5 & 3.5 & 5.4 \\
\hline Systolic blood pressure - high ${ }^{\mathrm{b}}$ & 0.5 & 0.6 & 0.9 & 2.0 & 1.1 & 0.5 & 1.0 & 1.1 \\
\hline Systolic blood pressure - low & 0.9 & 0.5 & 1.4 & 1.1 & I.I & 0.9 & 0.6 & I.I \\
\hline Diastolic blood pressure - high & 1.6 & 0.6 & 0.6 & 0.9 & 0.5 & 0.7 & 1.6 & 1.0 \\
\hline Diastolic blood pressure - lowe & 0.5 & 0.5 & 1.0 & I.I & 0.9 & 0.3 & 0.4 & 0.6 \\
\hline Pulse rate - high $^{f}$ & 0.2 & 0.2 & 0.3 & 0.4 & 0.4 & 0.1 & 0.1 & 0.3 \\
\hline Pulse rate - low $^{g}$ & 1.3 & 0.6 & 0.5 & 0.2 & 0.4 & 0.3 & 0.7 & 1.1 \\
\hline \multicolumn{9}{|l|}{ QTc interval } \\
\hline$>450 \mathrm{~ms}$ (males) or $470 \mathrm{~ms}$ (females) & 4.0 & 2.7 & 4.0 & 4.6 & 3.4 & 1.0 & 3.4 & 2.7 \\
\hline$>500 \mathrm{~ms}$ & 0 & 0.1 & 0 & 0 & 0 & 0 & 0.2 & 0 \\
\hline Increase from baseline $30-60 \mathrm{~ms}$ & 7.1 & 6.0 & 7.5 & 6.9 & 6.5 & 4.7 & 6.2 & 5.3 \\
\hline Increase from baseline $>60 \mathrm{~ms}$ & 0 & 0.1 & 0.1 & 0.2 & 0 & 0.3 & 0.1 & 0.3 \\
\hline
\end{tabular}

Notes: Measured on Day I and after 12 weeks of treatment in one study, on Day I and after 2 and 12 weeks in five studies, on Day I and after 4 and 12 weeks in two studies, and on Day I and after 4, 8, and 12 weeks in two studies; one study measured ECG and vital signs on Day I and Weeks 2 and I2 and laboratory evaluations on Weeks 4 and I2; ${ }^{b} \geq 180 \mathrm{mmHg}$ and increase from baseline by $\geq 20 \mathrm{mmHg}$, or $>200 \mathrm{mmHg} ;{ }^{c}<75 \mathrm{mmHg}$, or $\leq 90 \mathrm{mmHg}$ and decrease from baseline by $\geq 20 \mathrm{mmHg} ;{ }^{d} \geq 105$ $\mathrm{mmHg}$ and increase from baseline by $\geq 15 \mathrm{mmHg}$, or $>115 \mathrm{mmHg} ;{ }^{e}<40 \mathrm{mmHg}$, or $\leq 50 \mathrm{mmHg}$ and decrease from baseline by $\geq 15 \mathrm{mmHg} ;{ }^{f} \geq 120 \mathrm{bpm}$ and increase from baseline by $\geq 15 \mathrm{bpm}$, or $>130 \mathrm{bpm} ; 8<40 \mathrm{bpm}$, or $\leq 50 \mathrm{bpm}$ and decrease from baseline by $\geq 15 \mathrm{bpm}$.

Abbreviations: IND, indacaterol; FOR, formoterol; SLM, salmeterol; TIO, tiotropium; PBO, placebo; $n$, number.
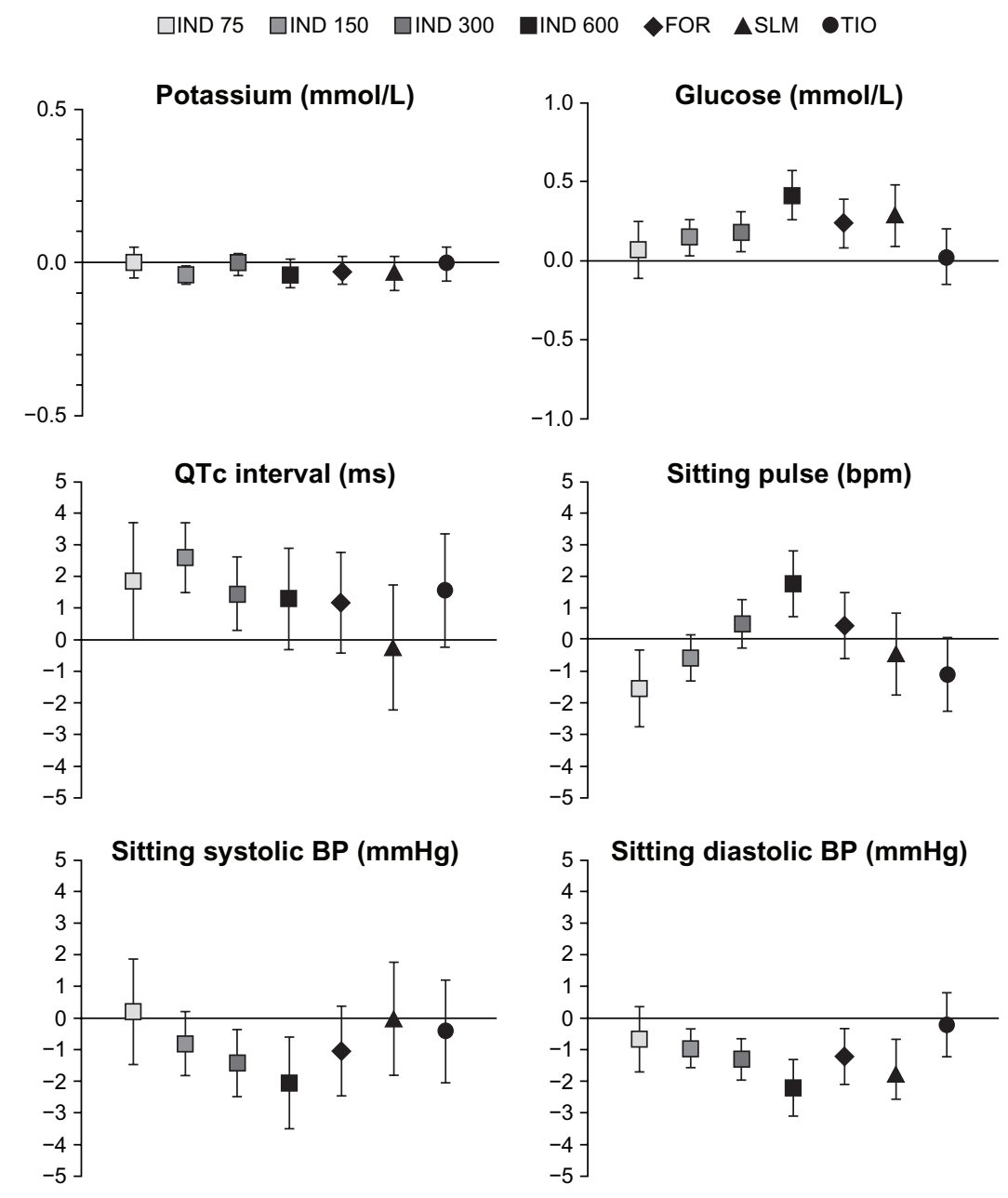

Figure 4 Mean $(95 \% \mathrm{Cl})$ differences from placebo in plasma potassium, blood glucose, QTc interval, sitting pulse and sitting systolic and diastolic blood pressure (BP) at I hour post-dose after 3 months of treatment.

Abbreviations: IND, indacaterol; FOR, formoterol; SLM, salmeterol; TIO, tiotropium; CI, confidence interval. 
pulse and blood pressure were clinically insignificant and no cause for concern. The cough following inhalation of indacaterol was quite common but was not associated with bronchospasm, increased withdrawal rate, or loss of efficacy, and should therefore not be considered a safety concern.

One limitation of this study is the limited numbers of patients on comparator therapies. For example, the nonsignificantly increased hazard ratios for MACE and death (Figures 1 and 2) with tiotropium should be viewed with caution. The FDA recently concluded that data did not support an increased risk of stroke, heart attack, or death associated with tiotropium dry powder inhaler. ${ }^{28}$ However, a recent meta-analysis did report an increased risk of mortality associated with the use of tiotropium taken via soft mist inhaler (a different formulation from that used in the studies included in this analysis). ${ }^{29}$ In the present analysis, tiotropium was administered open label in $35 \%$ of the patients in this treatment group, which may have introduced an element of bias. The results for the analysis of exacerbations may also be affected by low patient numbers in some of the treatment groups; elsewhere, for example, formoterol has been shown not to reduce COPD exacerbations. ${ }^{30,31}$ These results should also be interpreted in light of the overall low rates of exacerbations in all the studies included in this analysis.

The two doses of indacaterol of 150 and $300 \mu \mathrm{g}$, as licensed in many countries, provided the majority of the indacaterol data for this analysis and the results in these two treatment groups are therefore the best guide for potential safety and tolerability. Overall, the results with indacaterol 150 and $300 \mu \mathrm{g}$ demonstrate a good profile of safety and tolerability that is comparable with the other evaluated longacting bronchodilators. Compared with these two doses, results with the $75 \mu \mathrm{g}$ dose of indacaterol were occasionally anomalous but must be considered in the context that these data are less robust in terms of shorter length of treatment (maximum 12 weeks) and fewer patients exposed to this dose. However, in the two studies evaluating the $75 \mu \mathrm{g}$ dose, safety and tolerability profiles were similar to placebo. ${ }^{12}$ The results with the higher dose of indacaterol, $600 \mu \mathrm{g}$ given for 1 year, ${ }^{3}$ demonstrate a satisfactory therapeutic index between efficacy and safety with indacaterol.

In conclusion, this study finds that indacaterol is not associated with any significant safety concerns. Physicians choosing to prescribe indacaterol as regular maintenance treatment for patients with moderate-to-severe COPD in the expectation of bronchodilation and improved clinical outcomes may also be reassured of its good safety and tolerability profile.

\section{Disclosure}

JFD has received fees for consultancy and advisory work from Almirall, Boehringer-Ingelheim, GSK, Pfizer, Forest, Novartis, Sunovion, and Dey, and has received grant support from Boehringer-Ingelheim, Pfizer, Novartis, and Almirall.

DS has received lecture fees, support for conference attendance, advisory board fees, and research grants from pharmaceutical companies including GSK, Chiesi, AstraZeneca, CIPLA, Novartis, Forest, MSD, Boehringer-Ingelheim, and Almirall.

OK has received fees for consultancy and advisory work from Boehringer-Ingelheim and Novartis and, as a speaker, from Boehringer-Ingelheim, AstraZeneca, Novartis, and Meda. He has been an investigator in clinical studies for Almirall, Boehringer-Ingelheim, GSK, Novartis, Mundipharma, Bayer, Merck, Johnson and Johnson, and AstraZeneca.

DL, CL, and BK are employees of Novartis, which funded the studies providing the data and this analysis.

\section{Statement of authorship}

DL, CL, and BK made substantial contributions to the conception and design of the studies providing the data for the analysis described in this manuscript. JFD, DS, and $\mathrm{OK}$ were involved in the acquisition of data in one or more of the original studies. DL was responsible for the analysis of the data. All authors were involved in the concept and design of this article and the interpretation of the data, and had full access to the original data. All authors critically revised the article for important intellectual content and gave their final approval of the version to be published.

\section{Acknowledgments}

The authors thank the patients and investigators involved in the studies providing the data for this analysis. The authors were assisted by Sarah Filcek (ACUMED), a professional medical writer funded by Novartis, and David Young (Novartis).

\section{References}

1. Global Strategy for the Diagnosis, Management and Prevention of COPD, Global Initiative for Chronic Obstructive Lung Disease (GOLD) 2010. Available from: http://www.goldcopd.org. Accessed March 3, 2011.

2. Feldman G, Siler T, Prasad N, et al; INLIGHT 1 study group. Efficacy and safety of indacaterol $150 \mu \mathrm{g}$ once-daily in COPD: a double-blind, randomised, 12-week study. BMC Pulm Med. 2010;10:11. 
3. Dahl R, Chung KF, Buhl R, et al; INVOLVE (INdacaterol: Value in COPD: Longer Term Validation of Efficacy and Safety) Study Investigators. Efficacy of a new once-daily long-acting inhaled $\beta_{2}$-agonist indacaterol versus twice-daily formoterol in COPD. Thorax. 2010;65(6):473-479.

4. Donohue JF, Fogarty C, Lötvall J, et al; INHANCE Study Investigators. Once-daily bronchodilators for chronic obstructive pulmonary disease: indacaterol versus tiotropium. Am J Respir Crit Care Med. 2010;182(2): $155-162$.

5. Kornmann O, Dahl R, Centanni S, et al; INLIGHT-2 (Indacaterol Efficacy Evaluation Using 150- $\mu$ g Doses with COPD Patients) Study Investigators. Once-daily indacaterol versus twice-daily salmeterol for COPD: a placebo-controlled comparison. Eur Respir J. 2011; 37(2):273-279.

6. Chapman KR, Rennard SI, Dogra A, Owen R, Lassen C, Kramer B. Long-term safety and efficacy of indacaterol, a novel long-acting $\beta_{2}$-agonist, in subjects with COPD: a randomized, placebo-controlled study. Chest. 2011;140(1):68-75.

7. Feary JR, Rodrigues LC, Smith CJ, Hubbard RB, Gibson JE. Prevalence of major comorbidities in subjects with COPD and incidence of myocardial infarction and stroke: a comprehensive analysis using data from primary care. Thorax. 2010;65(11):956-962.

8. Sinden NJ, Stockley RA. Systemic inflammation and comorbidity in COPD: a result of "overspill" of inflammatory mediators from the lungs? Review of the evidence. Thorax. 2010;65(10):930-936.

9. Sharafkhaneh A, Petersen NJ, Yu HJ, Dalal AA, Johnson ML, Hanania NA. Burden of COPD in a government health care system: a retrospective observational study using data from the US Veterans Affairs population. Int J Chron Obstruct Pulmon Dis. 2010;5: $125-132$.

10. Korn S, Kerwin E, Atis S, Amos C, Owen R, Lassen C; on behalf of the INSIST study group. Indacaterol once-daily provides superior efficacy to salmeterol twice-daily in COPD: A 12-week study. Respir Med. 2011;105(5):719-726.

11. Buhl R, Dunn LJ, Disdier C, et al; on behalf of the INTENSITY study investigators. Blinded 12-week comparison of once-daily indacaterol and tiotropium in COPD. Eur Respir J. 2011;May 26. [Epub ahead of print.]

12. Kerwin EM, Meli J, Henley M, Lassen C, Kramer B. Efficacy and safety of indacaterol $75 \mu \mathrm{g}$ once daily in patients with moderateto-severe COPD. Am J Respir Crit Care Med. 2011;183:A1595 (American Thoracic Society International Conference Issue) [abstract].

13. Kinoshita M, Lee S-H, Hang L-W, et al. Efficacy and safety of indacaterol 150 and $300 \mu \mathrm{g}$ in Asian COPD patients. Am J Respir Crit Care Med. 2011;183:A1593 (American Thoracic Society International Conference Issue) [abstract].

14. Barnes PJ, Pocock SJ, Magnussen H, et al. Integrating indacaterol dose selection in a clinical study in COPD using an adaptive seamless design. Pulm Pharmacol Ther. 2010;23(3):165-171.

15. Mahler DA, D’Urzo A, Peckitt C, Lassen C, Kramer B. Combining once-daily bronchodilators in COPD: indacaterol plus tiotropium versus tiotropium alone. Am J Respir Crit Care Med. 2011;183:A1591 (American Thoracic Society International Conference Issue) [abstract].

16. US Department of Health and Human Services, Food and Drug Administration, Center for Drug Evaluation and Research (CDER). Guidance for Industry. Diabetes Mellitus - Evaluating Cardiovascular Risk in New Antidiabetic Therapies to Treat Type 2 Diabetes. December 2008. Available at: http://www.fda.gov/downloads/Drugs/ GuidanceComplianceRegulatoryInformation/Guidances/ucm071627. pdf. Accessed May 23, 2011.
17. US Department of Health and Human Services, Food and Drug Administration, Center for Drug Evaluation and Research (CDER). NDA 22-383 Briefing Document. Available at: http://www.fda.gov/ downloads/AdvisoryCommittees/CommitteesMeetingMaterials/ Drugs/Pulmonary-AllergyDrugsAdvisoryCommittee/UCM245639. pdf. Accessed 8 June, 2011.

18. Lulich KM, Goldie RG, Ryan G, Paterson JW. Adverse reactions to beta 2-agonist bronchodilators. Med Toxicol. 1986;1(4):286-299.

19. Barr RG, Celli BR, Mannino DM, et al. Comorbidities, patient knowledge, and disease management in a national sample of patients with COPD. Am J Med. 2009;122(4):348-355.

20. Salpeter SR, Buckley NS, Ormiston TM, Salpeter EE. Meta-analysis: effect of long-acting beta-agonists on severe asthma exacerbations and asthma-related deaths. Ann Intern Med. 2006;144(12):904-912.

21. Nelson HS, Weiss ST, Bleecker ER, Yancey SW, Dorinsky PM; SMART Study Group. The Salmeterol Multicenter Asthma Research Trial: a comparison of usual pharmacotherapy for asthma or usual pharmacotherapy plus salmeterol. Chest. 2006;129:15-26. Erratum in: Chest. 2006;129(1):1393.

22. Weatherall M, Wijesinghe M, Perrin K, Harwood M, Beasley R. Meta-analysis of the risk of mortality with salmeterol and the effect of concomitant corticosteroid therapy. Thorax. 2010;65(1):39-43.

23. Worth H, Chung KF, Felser JM, Hu H, Rueegg P. Cardio- and cerebrovascular safety of indacaterol vs formoterol, salmeterol, tiotropium and placebo in COPD. Respir Med. 2011;105(4):571-579.

24. Calverley PM, Anderson JA, Celli B, et al; TORCH Investigators. Cardiovascular events in patients with COPD: TORCH study results. Thorax. 2010;65(8):719-725.

25. Calverley PM, Anderson JA, Celli B, et al; TORCH investigators. Salmeterol and fluticasone propionate and survival in chronic obstructive pulmonary disease. N Engl J Med. 2007;356(8):775-789.

26. Kliber A, Lynd LD, Sin DD. The effects of long-acting bronchodilators on total mortality in patients with stable chronic obstructive pulmonary disease. Respir Res. 2010;11:56.

27. Lee TA, Pickard AS, Au DH, Bartle B, Weiss KB. Risk for death associated with medications for recently diagnosed chronic obstructive pulmonary disease. Ann Intern Med. 2008;149(6):380-390.

28. Michele TM, Pinheiro S, Iyasu S. The safety of tiotropium-the FDA's conclusions. N Engl J Med. 2010;363(12):1097-1099.

29. Singh S, Loke YK, Enright PL, Furberg CD. Mortality associated with tiotropium mist inhaler in patients with chronic obstructive pulmonary disease: systematic review and meta-analysis of randomised controlled trials. BMJ. 2011;342:d3215. doi: 10.1136/bmj.d3215.

30. Szafranski W, Cukier A, Ramirez A, et al. Efficacy and safety of budesonide/formoterol in the management of chronic obstructive pulmonary disease. Eur Respir J. 2003;21(1):74-81. Erratum in: Eur Respir J. 2003;21(5):912.

31. Calverley PM, Boonsawat W, Cseke Z, Zhong N, Peterson S, Olsson H. Maintenance therapy with budesonide and formoterol in chronic obstructive pulmonary disease. Eur Respir J. 2003;22:912-919. Erratum in: Eur Respir J. 2004;24(6):1075. 


\section{Supplementary tables}

Table SI Details of the studies included in the safety evaluation

\begin{tabular}{|c|c|c|c|c|}
\hline Length (weeks) & Patients (n) & Treatments & ClinicalTrials.gov reference & Reference \\
\hline \multirow[t]{4}{*}{52} & 1732 & Indacaterol $300 \mu \mathrm{g}$ od & NCT00393458 & 3 \\
\hline & & Indacaterol $600 \mu \mathrm{g}$ od & & \\
\hline & & Placebo & & \\
\hline & & Formoterol $12 \mu \mathrm{g}$ bid & & \\
\hline \multirow[t]{4}{*}{26} & 1683 & Indacaterol $150 \mu \mathrm{g}$ od & NCT00463567 & $4^{\mathrm{a}}$ \\
\hline & & Indacaterol $300 \mu \mathrm{g}$ od & & \\
\hline & & Placebo & & \\
\hline & & Open-label tiotropium $18 \mu \mathrm{g}$ od & & \\
\hline \multirow[t]{3}{*}{52} & 415 & Indacaterol $150 \mu \mathrm{g}$ od & NСT00677807 & $6^{\mathrm{a}}$ \\
\hline & & Indacaterol $300 \mu \mathrm{g}$ od & & \\
\hline & & Placebo & & \\
\hline \multirow[t]{3}{*}{26} & 1002 & Indacaterol $150 \mu \mathrm{g}$ od & NCT00567996 & 5 \\
\hline & & Salmeterol $50 \mu \mathrm{g}$ bid & & \\
\hline & & Placebo & & \\
\hline \multirow[t]{2}{*}{12} & 416 & Indacaterol I $50 \mu \mathrm{g}$ od & NCT00624286 & 2 \\
\hline & & Placebo & & \\
\hline \multirow[t]{2}{*}{12} & 1123 & Indacaterol I $50 \mu \mathrm{g}$ od & NСT0082I093 & 10 \\
\hline & & Salmeterol $50 \mu \mathrm{g}$ bid & & \\
\hline \multirow[t]{2}{*}{12} & 1598 & Indacaterol I $50 \mu \mathrm{g}$ od & NСT0090073I & 11 \\
\hline & & Tiotropium $18 \mu \mathrm{g}$ od & & \\
\hline \multirow[t]{2}{*}{12} & 323 & Indacaterol $75 \mu \mathrm{g}$ od & NCT0I072448 & 12 \\
\hline & & Placebo & & \\
\hline \multirow[t]{2}{*}{12} & 318 & Indacaterol $75 \mu \mathrm{g}$ od & NCT0I068600 & 12 \\
\hline & & Placebo & & \\
\hline \multirow[t]{3}{*}{12} & 347 & Indacaterol I $50 \mu \mathrm{g}$ od & NCT00794I57 & 13 \\
\hline & & Indacaterol $300 \mu \mathrm{g}$ od & & \\
\hline & & Placebo & & \\
\hline \multirow[t]{3}{*}{26} & 563 & Indacaterol $150 \mu \mathrm{g}$ od & NCT00792805 & - \\
\hline & & Indacaterol $300 \mu \mathrm{g}$ od & & \\
\hline & & Placebo & & \\
\hline
\end{tabular}

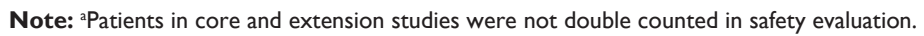

Abbreviations: od, once daily; bid, twice daily; n, number.

Table S2 Withdrawal rates and reasons for withdrawal

\begin{tabular}{|c|c|c|c|c|c|c|c|c|}
\hline & $\begin{array}{l}\text { IND } 75 \\
\mathrm{n}=449\end{array}$ & $\begin{array}{l}\text { IND I50 } \\
n=2611\end{array}$ & $\begin{array}{l}\text { IND } 300 \\
\mathrm{n}=11 \mathrm{I57}\end{array}$ & $\begin{array}{l}\text { IND } 600 \\
n=547\end{array}$ & $\begin{array}{l}\text { FOR } \\
n=556\end{array}$ & $\begin{array}{l}\text { SLM } \\
\mathrm{n}=\mathbf{8 9 5}\end{array}$ & $\begin{array}{l}\text { TIO } \\
n=1214\end{array}$ & $\begin{array}{l}\text { PBO } \\
n=2012\end{array}$ \\
\hline Total withdrawals & $50(I I . I)$ & $311(11.9)$ & $221(19.1)$ & $119(21.8)$ & $|2|(2 \mid .8)$ & $88(9.8)$ & | 43 (I I.8) & $475(23.6)$ \\
\hline Adverse event & $22(4.9)$ & $123(4.7)$ & $73(6.3)$ & $34(6.2)$ & $44(7.9)$ & $28(3.1)$ & $44(3.6)$ & $134(6.7)$ \\
\hline Withdrawal of consent & $15(3.3)$ & $75(2.9)$ & $58(5.0)$ & $43(7.9)$ & $34(6.1)$ & $24(2.7)$ & $27(2.2)$ & $140(7.0)$ \\
\hline $\begin{array}{l}\text { Unsatisfactory therapeutic } \\
\text { effect }\end{array}$ & $2(0.5)$ & II (0.4) & $27(2.3)$ & $9(1.7)$ & $13(2.3)$ & $4(0.5)$ & $12(1.0)$ & $85(4.2)$ \\
\hline Protocol deviation & $5(1.1)$ & $40(1.5)$ & $25(2.2)$ & $12(2.2)$ & $13(2.3)$ & $17(1.9)$ & $25(2.1)$ & $5 \mathrm{I}(2.5)$ \\
\hline Administrative problems & 0 & $10(0.4)$ & $16(1.4)$ & $9(1.7)$ & $6(1.1)$ & $2(0.2)$ & $4(0.3)$ & $15(0.8)$ \\
\hline Loss to follow-up & $3(0.7)$ & $33(1.3)$ & $14(1.2)$ & $7(1.3)$ & $6(1.1)$ & $8(0.9)$ & $16(1.3)$ & $27(1.3)$ \\
\hline Abnormal test procedure result & I $(0.2)$ & $8(0.3)$ & $4(0.4)$ & $3(0.6)$ & $\mathrm{I}(0.2)$ & $2(0.2)$ & $5(0.4)$ & $5(0.3)$ \\
\hline Abnormal laboratory value & $2(0.5)$ & $7(0.3)$ & $2(0.2)$ & I $(0.2)$ & 0 & $\mathrm{I}(0 . \mathrm{I})$ & $6(0.5)$ & $5(0.3)$ \\
\hline Death & 0 & $4(0.2)$ & $2(0.2)$ & 0 & $4(0.7)$ & $\mathrm{I}(0 . \mathrm{I})$ & $4(0.3)$ & II (0.6) \\
\hline $\begin{array}{l}\text { No further requirement for } \\
\text { study drug }\end{array}$ & 0 & 0 & 0 & I $(0.2)$ & 0 & 0 & 0 & $\mathrm{I}(0 . \mathrm{I})$ \\
\hline Not stated & 0 & 0 & 0 & 0 & 0 & 0 & 0 & $\mathrm{I}(0.1)$ \\
\hline Inability to use the inhaler & 0 & 0 & 0 & 0 & 0 & $\mathrm{I}(0 . \mathrm{I})$ & 0 & 0 \\
\hline
\end{tabular}

Note: Data are $\mathrm{n}(\%)$.

Abbreviations: IND, indacaterol; FOR, formoterol; SLM, salmeterol; TIO, tiotropium; PBO, placebo; n, number. 
Table S3 Preferred terms in the broad major adverse cardiovascular events (MACE) category

\begin{tabular}{|c|c|c|}
\hline \multicolumn{3}{|c|}{ Central nervous system hemorrhages and cerebrovascular conditions* } \\
\hline Agnosia & Hematomyelia & Cerebellar ischemia \\
\hline Amaurosis fugax & Hemorrhage intracranial & Cerebral arteriosclerosis \\
\hline Angiogram cerebral abnormal & Hemorrhagic cerebral infarction & Cerebral artery embolism \\
\hline Aphasia & Hemorrhagic stroke & Cerebral artery occlusion \\
\hline Balint's syndrome & Hemorrhagic transformation stroke & Cerebral artery stenosis \\
\hline Carotid artery aneurysm & Intracerebral hematoma evacuation & Cerebral artery thrombosis \\
\hline Carotid artery dissection & Intracranial hematoma & Cerebral infarction \\
\hline Central pain syndrome & Intraventricular hemorrhage & Cerebral infarction fetal \\
\hline Cerebral aneurysm ruptured syphilitic & Intraventricular hemorrhage neonatal & Cerebral ischemia \\
\hline Cerebrovascular accident prophylaxis & Meningorrhagia & Cerebral revascularization synangiosis \\
\hline Charcot-Bouchard microaneurysms & Putamen hemorrhage & Cerebral thrombosis \\
\hline Diplegia & Ruptured cerebral aneurysm & Cerebral vasoconstriction \\
\hline Dysarthria & Spinal cord hemorrhage & Cerebral venous thrombosis \\
\hline Hemiparesis & Spinal epidural hemorrhage & Cerebrovascular accident \\
\hline Hemiplegia & Spinal hematoma & Cerebrovascular disorder \\
\hline Intra-cerebral aneurysm operation & Stroke in evolution & Cerebrovascular insufficiency \\
\hline Intracranial aneurysm & Subarachnoid hemorrhage & Cerebrovascular spasm \\
\hline Monoparesis & Subarachnoid hemorrhage neonatal & Cerebrovascular stenosis \\
\hline Monoplegia & Subdural hemorrhage & Embolic cerebral infarction \\
\hline Paralysis & Subdural hemorrhage neonatal & Embolic stroke \\
\hline Paralysis flaccid & Thalamus hemorrhage & Ischemic cerebral infarction \\
\hline Paraparesis & Basal ganglia infarction & Ischemic stroke \\
\hline Paraplegia & Basilar artery occlusion & Lacunar infarction \\
\hline Paresis & Basilar artery stenosis & Lateral medullary syndrome \\
\hline Quadriparesis & Basilar artery thrombosis & Millard-Gubler syndrome \\
\hline Quadriplegia & Brain stem infarction & Moyamoya disease \\
\hline Red blood cells CSF positive & Brain stem ischemia & Post procedural stroke \\
\hline Spastic paralysis & Brain stem stroke & Precerebral artery occlusion \\
\hline Spastic paraplegia & Brain stem thrombosis & Reversible ischemic neurological deficit \\
\hline Visual midline shift syndrome & Capsular warning syndrome & Spinal artery embolism \\
\hline Basal ganglia hemorrhage & Carotid arterial embolus & Stroke in evolution \\
\hline Brain stem hemorrhage & Carotid arteriosclerosis & Thalamic infarction \\
\hline Brain stem stroke & Carotid artery bypass & Thrombotic cerebral infarction \\
\hline Carotid aneurysm rupture & Carotid artery disease & Thrombotic stroke \\
\hline Cerebellar hematoma & Carotid artery insufficiency & Transient ischemic attack \\
\hline Cerebellar hemorrhage & Carotid artery occlusion & Vascular encephalopathy \\
\hline Cerebral arteriovenous malformation hemorrhagic & Carotid artery stenosis & Vertebral artery occlusion \\
\hline Cerebral hematoma & Carotid artery stent insertion & Vertebral artery stenosis \\
\hline Cerebral hemorrhage & Carotid artery thrombosis & Vertebral artery thrombosis \\
\hline Cerebral hemorrhage fetal & Carotid endarterectomy & Vertebrobasilar insufficiency \\
\hline Cerebral hemorrhage neonatal & Cerebellar artery occlusion & Wallenberg syndrome \\
\hline Cerebral microhemorrhage & Cerebellar artery thrombosis & Brachiocephalic artery occlusion \\
\hline Cerebrovascular accident & Cerebellar embolism & Carotid artery stent removal \\
\hline Cerebrovascular disorder & Cerebellar infarction & \\
\hline \multicolumn{3}{|l|}{ Myocardial infarction* } \\
\hline Acute coronary syndrome & Coronary artery thrombosis & Papillary muscle infarction \\
\hline Acute myocardial infarction & Coronary bypass thrombosis & Post procedural myocardial infarction \\
\hline Blood creatine phosphokinase abnormal & Electrocardiogram $\mathrm{Q}$ wave abnormal & Postinfarction angina \\
\hline Blood creatine phosphokinase increased & Electrocardiogram ST segment abnormal & Scan myocardial perfusion abnormal \\
\hline Blood creatine phosphokinase MB abnormal & Electrocardiogram ST segment elevation & Silent myocardial infarction \\
\hline Blood creatine phosphokinase MB increased & Electrocardiogram ST-T segment elevation & Troponin I increased \\
\hline Cardiac enzymes increased & Infarction & Troponin increased \\
\hline Coronary artery embolism & Kounis syndrome & Troponin T increased \\
\hline Coronary artery occlusion & Myocardial infarction & Vascular graft occlusion \\
\hline Coronary artery reocclusion & Myocardial reperfusion injury & \\
\hline
\end{tabular}

Notes: *Level 2 standardized Medical Dictionary of Regulatory Activities (MedDRA) query (SMQs) (subgroups of the "ischemic heart disease" and "cerebrovascular disorders" Level I SMQs, respectively) from MedDRA version 13.0. 
Table S4 Preferred terms in the custom major adverse cardiovascular events (MACE) category

\begin{tabular}{lll}
\hline Acute myocardial infarction & Cerebrovascular accident & Moyamoya disease \\
Basilar artery thrombosis & Coronary artery thrombosis & Myocardial infarction \\
Brain stem infarction & Embolic cerebral infarction & Papillary muscle infarction \\
Brain stem stroke & Embolic stroke & Post procedural myocardial \\
Brain stem thrombosis & Hemorrhagic cerebral infarction & Post procedural stroke \\
Carotid arterial embolus & Hemorrhagic stroke & Silent myocardial infarction \\
Carotid artery thrombosis & Hemorrhagic transformation stroke & Stroke in evolution \\
Cerebellar infarction & Ischemic cerebral infarction & Thalamic infarction \\
Cerebral artery embolism & Ischemic stroke & Thrombotic cerebral infarction \\
Cerebral artery thrombosis & Lacunar infarction & Thrombotic stroke \\
Cerebral infarction & Cerebrovascular accident & Wallenberg syndrome \\
Cerebral thrombosis & Lateral medullary syndrome & \\
\hline
\end{tabular}

\section{Publish your work in this journal}

The International Journal of COPD is an international, peer-reviewed journal of therapeutics and pharmacology focusing on concise rapid reporting of clinical studies and reviews in COPD. Special focus is given to the pathophysiological processes underlying the disease, intervention programs, patient focused education, and self management protocols.
This journal is indexed on PubMed Central, MedLine and CAS. The manuscript management system is completely online and includes a very quick and fair peer-review system, which is all easy to use. Visit $\mathrm{http} / / / \mathrm{www}$.dovepress.com/testimonials.php to read real quotes from published authors.

Submit your manuscript here: http://www.dovepress.com/international-journal-of-copd-journal 